\title{
1 Brain expression quantitative trait locus and network 2 analysis reveals downstream effects and putative 3 drivers for brain-related diseases
}

Niek de Klein ${ }^{1,4,6,10}$, Ellen A. Tsai ${ }^{2,10}$, Martijn Vochteloo ${ }^{1,3,10}$, Denis Baird ${ }^{2,9,10}$, Yunfeng Huang ${ }^{2}$, Chia-Yen Chen ${ }^{2}$, Sipko van Dam ${ }^{1,5}$, Patrick Deelen ${ }^{1}$, Olivier B. Bakker ${ }^{1}$, Omar El Garwany ${ }^{1,6}$, Zhengyu Ouyang ${ }^{7}$, Eric E. Marshall ${ }^{2}$, Maria I. Zavodszky ${ }^{2}$, Wouter van Rheenen ${ }^{8}$, Mark K. Bakker $^{8}$, Jan Veldink ${ }^{8}$, Tom R. Gaunt ${ }^{9}$, Heiko Runz ${ }^{2,12}$, Lude Franke ${ }^{1,4,12}$, Harm-Jan Westra ${ }^{1,4,12}$

1. Department of Genetics, University Medical Center Groningen, University of Groningen, Hanzeplein 1, Groningen, The Netherlands

2. Translational Biology, Research \& Development, Biogen Inc., 225 Broadway, Cambridge, MA, USA

3. Institute for Life Science \& Technology, Hanze University of Applied Sciences, Zernikeplein 11, 9747 AS Groningen, The Netherlands

4. Oncode Investigator

5. Ancora Health, Herestraat 106, 9711 LM, Groningen, The Netherlands

6. Wellcome Sanger Institute, Wellcome Genome Campus, Hinxton, UK

7. BioInfoRx, Inc., 510 Charmany Dr, Suite 275A, Madison, WI 53719, USA

8. Department of Neurology, UMC Utrecht Brain Center, University Medical Center Utrecht, Utrecht University, Utrecht, The Netherlands.

9. MRC Integrative Epidemiology Unit, Bristol Medical School, University of Bristol, Bristol, UK

10. These authors contributed equally

11. These authors contributed equally

12. These authors jointly supervised the work 


\section{Abstract}

29 Gaining insight into the downstream consequences of non-coding variants is an essential step

30 towards the identification of therapeutic targets from genome-wide association study (GWAS)

31 findings. Here we have harmonized and integrated 8,727 RNA-seq samples with accompanying

32 genotype data from multiple brain-regions from 14 datasets. This sample size enabled us to

33 perform both cis- and trans-expression quantitative locus (eQTL) mapping. Upon comparing the

34 brain cortex cis-eQTLs (for 12,307 unique genes at FDR $<0.05$ ) with a large blood cis-eQTL

35 analysis ( $n=31,684$ samples), we observed that brain eQTLs are more tissue specific than

36 previously assumed.

38 We inferred the brain cell type for 1,515 cis-eQTLs by using cell type proportion information.

39 We conducted Mendelian Randomization on 31 brain-related traits using cis-eQTLs as

40 instruments and found 159 significant findings that also passed colocalization. Furthermore, two

41 multiple sclerosis (MS) findings had cell type specific signals, a neuron-specific cis-eQTL for

$42 \quad C Y P 24 A 1$ and a macrophage specific cis-eQTL for CLECL1.

44 To further interpret GWAS hits, we performed trans-eQTL analysis. We identified 2,589 trans-

45 eQTLs (at FDR <0.05) for 373 unique SNPs, affecting 1,263 unique genes, and 21 replicated

46 significantly using single-nucleus RNA-seq data from excitatory neurons.

48 We also generated a brain-specific gene-coregulation network that we used to predict which

49 genes have brain-specific functions, and to perform a novel network analysis of Alzheimer's

50 disease (AD), amyotrophic lateral sclerosis (ALS), multiple sclerosis (MS) and Parkinson's 
51 disease (PD) GWAS data. This resulted in the identification of distinct sets of genes that show

52 significantly enriched co-regulation with genes inside the associated GWAS loci, and which

53 might reflect drivers of these diseases.

\section{Introduction}

56 Diseases of the brain manifesting as psychiatric or neurological conditions continue to be a

57 massive global health burden: The World Health Organization estimates that in 2019 globally

58280 million individuals were affected by depression, 39.5 million by bipolar disorder, and 287.4

59 million by schizophrenia ${ }^{1}$. Likewise, the fraction of 50 million people living with dementia today

60 is expected to rise to 152 million by $2050^{2}$, with similar trajectories for other neurodegenerative

61 diseases. While substantial progress has been made in uncovering the genetic basis of psychiatric

62 and neurological diseases through genome-wide association studies (GWAS), much of how the

63 identified genetic variants impact brain function is still unknown.

65 To translate from genetic signals to mechanisms, associations with gene expression levels, or 66 expression quantitative trait loci (eQTL) have shown great potential. eQTLs can be divided in

67 direct effects of local genetic variants (cis-eQTLs) and indirect effects of distal variants (trans-

68 eQTLs). Cis-eQTLs and trans-eQTLs can aid interpretation of GWAS loci in several ways. Cis-

69 eQTLs aid interpretation by identifying direct links between genes and phenotypes through

70 causal inference approaches such as Mendelian randomization (MR) instrumented on QTLs and

71 genetic colocalization analysis, whereas trans-eQTLs expose sets of downstream genes and

72 pathways on which the effects of disease variants converge. 
74 eQTLs are dynamic features and vary with tissue, cell type and additional factors such as

75 response to stimulation. For an optimal interrogation of GWAS loci, it is therefore desirable to

76 perform eQTL analyses in disease-relevant tissues ${ }^{3}$. To help interpret GWAS of

77 neurodegenerative and psychiatric diseases, several brain-derived eQTL studies have been

78 published, including meta-analyses by the PsychENCODE ${ }^{4}$ and $\mathrm{AMP}-\mathrm{AD}^{5}$ consortia, which

79 cover 1,866 and 1,433 individuals, respectively. However, to yield reliable results, statistical

80 approaches such as MR and colocalization require robust effect size estimates from even larger

81 carefully curated eQTL datasets. Large sample sizes are better suited to decompose eQTL effects

82 to specific cell types.

84 To maximize the potential of eQTL-based analyses in brain, we here combined and rigorously

85 harmonized brain RNA-seq and genotype data from 15 different cohorts, including 8,727 RNA-

86 seq samples from all major brain eQTL studies and publicly available samples from the

87 European Nucleotide Archive (ENA). By leveraging the statistical power across these datasets,

88 we created a gene coregulation network based on 8,544 RNA-seq samples covering different

89 brain regions and performed cis- and trans-eQTL analysis in up to 2,970 individuals of European

90 descent, with replication in up to 420 individuals of African descent. This sample size enabled us

91 to make inferences on the brain cell types in which eQTLs operate, and to systematically conduct

92 Mendelian Randomization and colocalization analyses to find shared genetic effects between

93 eQTLs and GWAS traits. This prioritized likely causal genes from GWAS loci for 31 brain-

94 related traits, including neurodegenerative and psychiatric conditions. Additionally, this

95 identified cell type dependent eQTLs that may be associated with disease risk (Figure 1). 


\section{Results}

98 Leveraging public RNA-seq and genotype data to create large, harmonized brain

\section{9 eQTL and gene co-regulation datasets}

100 We combined 15 eQTL datasets into the 'MetaBrain' resource to maximize statistical power to

101 detect eQTLs and to create a brain specific gene coregulation network (Figure 2;

102 Supplementary Table 1, Supplementary Figures 1-5). MetaBrain includes 7,604 RNA-seq

103 samples and accompanying genotypes from the AMP-AD consortium ${ }^{6}$ (AMP-AD MAYO ${ }^{6}$,

$104 \operatorname{ROSMAP}^{6}$ and MSBB $\left.{ }^{6}\right)$, Braineac $^{7}$, the PsychENCODE consortium ${ }^{8}\left(\right.$ Bipseq $^{4}$, BrainGVEX $^{4}$,

$105 \mathrm{CMC}^{9}$, GVEX and UCLA_ASD $\left.{ }^{4}\right)$, BrainSeq $^{10}, \mathrm{NABEC}^{11}$, $\operatorname{TargetALS}^{12}$, and GTEx ${ }^{3}$.

106 Additionally, we carefully selected 1,759 brain RNA-seq samples from the European Nucleotide

107 Archive (ENA) ${ }^{13}$, calling and imputing genotypes based on the RNA-seq alignment

108 (Supplementary Note, Supplementary Figure 1). There were 8,727 RNA-seq samples

109 remaining after realignment and stringent quality control (Methods and Supplementary Note,

110 Supplementary Figure 2-3). Using slightly different quality control measures, we created a

111 gene network using 8,544 samples (Supplementary Note). We corrected the RNA-seq data for

112 technical covariates and defined 7 major tissue groups (amygdala, basal ganglia, cerebellum,

113 cortex, hippocampus, hypothalamus and spinal cord): Principal Component Analysis (PCA) on

114 the RNA-seq data showed clear clustering by these major tissue groups, resembling brain

115 physiology (Figure 2D, Supplementary Figure 4). Genotype data revealed individuals from

116 different ethnicities (Figure 2B; Supplementary Figure 5), including 5,138 samples from

117 European descent (EUR) and 805 samples from African descent (AFR). We created 6 cis-eQTL

118 discovery datasets: Basal ganglia-EUR $(\mathrm{n}=208)$, Cerebellum-EUR $(\mathrm{n}=492)$, Cortex-EUR

$119(\mathrm{n}=2,970)$, Cortex-AFR $(\mathrm{n}=420)$, Hippocampus-EUR $(\mathrm{n}=168)$ and Spinal cord-EUR $(\mathrm{n}=108$; 
120 Supplementary Table 1, Figure 2C). Cis-eQTLs were not calculated for amygdala and

121 hypothalamus tissue groups due to the small sample size $(\mathrm{n}<100)$.

\section{$124 \quad 41 \%$ of the cortex cis-eQTL genes are regulated by multiple independent variants}

125 Within each discovery dataset, we performed a sample-size weighted cis-eQTL meta-analysis on 126 common variants (MAF>1\%), within 1 megabase $(\mathrm{Mb})$ of the transcription start site (TSS) of a 127 protein-coding gene. We identified 1,317 (Basal ganglia-EUR), 6,865 (Cerebellum-EUR), 5,440

128 (Cortex-AFR), 11,803 (Cortex-EUR), 990 (Hippocampus-EUR), and 811 (Spinal cord-EUR) cis129 eQTL genes (FDR<0.05; Figure 3A; Supplementary Table 2). Cis-eQTL effect directions were 130 highly concordant between datasets included in the Cortex-EUR meta-analysis (median 131 Spearman r=0.80; median allelic concordance=89\%; Supplementary Figure 6), indicating

132 robustness of the identified effects across datasets. We observed that significant $c i s$-eQTL

133 findings were sensitive to RNA-seq alignment strategies, and it is difficult to confidently

134 ascertain cis-eQTLs in regions with multiple haplotypes represented on patch chromosomes, like 135 the MAPT locus on 17q21 (Supplementary Note, Supplementary Figures 7-9). We next

136 performed conditional analysis to identify independent associations in each cis-eQTL locus (e.g.,

137 secondary, tertiary and quaternary eQTLs). In Cortex-EUR, 4,791 genes had a significant

138 secondary cis-eQTL (41\% of cis-eQTL genes identified in this dataset). 1,658 genes had tertiary

139 and 598 had quaternary cis-eQTLs. We also identified secondary associations for the other

140 discovery datasets albeit to a lesser extent (Figure 3A; Supplementary Table 2 and 3). 


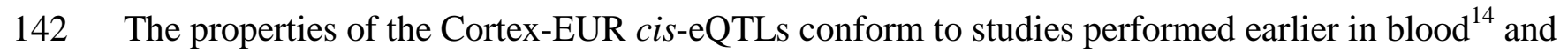

143 brain $^{15}$ (Figure 3B): primary lead cis-eQTL SNPs were generally located close (median distance:

14431 kilobase; $\mathrm{kb}$ ) to the transcription start site (TSS; Figure 3B) and cis-eQTL genes had a lower

145 probability for loss of function intolerance $\left(\mathrm{pLI} ; \chi^{2} \mathrm{p}=6.35 \times 10^{-147}\right)$. Genes with a cis-eQTL

146 generally had a higher median expression than those without (Wilcoxon p-value: 9.96x10 ${ }^{-12}$ ).

147 Contrary to blood, where genes in the highest expression decile are the most likely to have a cis-

148 eQTL, the third decile of gene expression had the most cis-eQTLs in cortex, and higher deciles

149 had increasingly lower proportions of eQTLs (Supplementary Note, Supplementary Figure

150 10A). This could suggest that highly expressed genes in the cortex have tighter genetic

151 regulation than highly expressed genes in the blood, although we did not observe differences

152 when comparing variance per gene expression decile between blood and brain (Supplementary

153 Note, Supplementary Figure 10B). Cortex-EUR cis-eQTL genes showed limited functional

154 enrichment for human phenotype ontologies (HPO), GO ontologies and TRANSFAC ${ }^{16}$

155 transcription factor motifs (Supplementary Figure 10C and D, Supplementary Table 4). We

156 observed similar patterns for secondary, tertiary and quaternary cis-eQTLs (Supplementary

157 Note).

159 We investigated differences in cis-eQTLs due to ancestry, brain region, data sets and tissue type.

160 We compared Cortex-EUR, Cortex-AFR and a smaller, East Asian cortex dataset (Cortex-EAS;

$161 \mathrm{n}=208$, limited to the ENA cohort; Figure 2C) and observed high concordance between the

162 different ethnicities (>95.67\%; Figure 3C). There was high concordance between different brain

163 regions overall (>94.58\%), though the cerebellum showed lower concordance with the cerebral

164 brain regions (Figure 3D). Despite the limited sample size compared to Cortex-EUR, we 
165 identified 846 cis-eQTLs that were unique to Cerebellum-EUR (Supplementary Figure 11A).

166 Of the 846 Cerebellum-EUR unique cis-eQTL genes, 184 had low gene expression levels in

167 cortex, which may explain why they did not have a cis-eQTL in that tissue (Supplementary

168 Figure 11B, C, Supplementary Note). For the remaining 662 genes that were highly expressed

169 in both cortex and cerebellum, we performed functional enrichment of transcription factor

170 binding sites (TFBS; Supplementary Table 5, Supplementary Note) and determined that these

171 genes were enriched for TFBS of 101 distinct transcription factors. Five of these transcription

172 factors had low gene expression in cortex and high expression in cerebellum (EOMES, TFAP2B,

173 TFAP2A, IRXI and IRX5, Supplementary Figure 11D). These transcription factors might

174 explain the difference in cis-eQTL genes found in cerebellum but not in cortex, while many of

175 these cis-eQTL genes are expressed in both tissues. Next, we compared Cortex-EUR cis-eQTLs

176 with different tissues from the GTEx project (Figure 3E; Supplementary Figure 12,

177 Supplementary Table 6). There was high concordance in brain-related tissues (cerebral tissues,

$178>98 \%$ and cerebellar tissues, >94\%) compared to other tissue types, and the lowest concordance

179 rates were observed in testis $(84 \%)$ and whole blood (85\%). We also compared Cortex-EUR cis-

180 eQTLs with eQTLGen ${ }^{17}$, a large blood-based eQTL dataset (n=31,684; majority EUR ancestry)

181 and observed a 76\% concordance rate (Supplementary Figure 13; Supplementary Table 7)

182 with a moderate correlation of $c i s$-eQTL effect sizes $\left(\mathrm{R}_{\mathrm{b}}=0.54\right.$ including all eQTLs, or $\mathrm{R}_{\mathrm{b}}=0.62$

183 when pruning genes within $1 \mathrm{Mb})^{18}$, supporting the lower concordance observed in GTEx-blood.

184 Since we found that $24 \%$ of the shared cis-eQTLs between blood and brain showed opposite

185 allelic effects, these results suggest that with larger sample sizes, more tissue specific regulatory

186 variants can be identified. If a causal tissue-specific regulatory variant resides on a haplotype that

187 also contains a variant that is specific for another tissue, it is well conceivable that opposite 
188 allelic effects are going to be observed when contrasting eQTLs for these two tissues ${ }^{19}$. Since the

189 procedures for eQTL mapping were identical between MetaBrain and eQTLGen, our results

190 highlight the relevance of tissue-specific eQTL mapping to accurately assess the directionality of

191 eQTLs, which can elucidate eQTLs with opposite allelic effects ${ }^{20}$. This direct comparison

192 illustrates the importance of investigating the appropriate tissue type for the interpretation of

193 GWAS signals.

\section{8\% of Cortex cis-eQTLs are mediated by cell type proportion differences}

196 Cell type dependent eQTLs can be identified in bulk RNA-seq data by performing cell type

197 deconvolution and determining cell type interaction eQTLs (ieQTLs) ${ }^{3,21,22}$. We predicted five

198 major cell types using single cell RNA-seq derived signature profiles ${ }^{23}$. Of these, neurons were

199 the most abundant cell type (median cell proportion: 32.8\%), followed by endothelial cells

200 (24.9\%), macrophages (17.8\%), oligodendrocytes (12.4\%) and astrocytes $(12.1 \%$;

201 Supplementary Figure 14). We predicted similar proportions for cerebellum as well as other

202 brain regions. We observed that predicted cell proportions are different for spinal cord, showing

203 a relatively low proportion of neuronal cells and high proportions of macrophage and

204 oligodendrocytes compared to other brain tissues, as was previously reported ${ }^{24}$ (Supplementary

205 Figures 15 and 16). Predicted neuron proportions in both cortex and cerebellum were negatively

206 correlated with the predicted proportions of other cell types, and predicted endothelial cell

207 proportions were negatively correlated with predicted macrophage proportions (Figure 4A).

208 Predicted cell type proportions were positively correlated with immunochemistry (IHC) counts

209 from the ROSMAP cohort $^{25}$, both overall (Spearman $\mathrm{r}=0.71$; Figure 4B) and per individual cell

210 type (Spearman $r>0.1$; Figure 4B). It is difficult to validate these cell type proportion predictions 
211 due to the small scale of the IHC experiment, but also because IHC and bulk RNA-seq reflect

212 different aspects of gene or protein expression. Thus, there is a level of uncertainty for the

213 expected proportion for each cell type ${ }^{26,27}$.

215 With these predicted cell type proportions, we used DeconQTL ${ }^{22}$ to identify interaction-eQTLs

216 (ieQTLs) by testing 18,850 cis-eQTLs in Cortex-EUR and 8,347 cis-eQTLs in cerebellum

217 (including primary, secondary, tertiary and quaternary eQTLs). We identified 1,515 significant

218 ieQTLs $(8 \%)$ in at least one cell type (Benjamini-Hochberg; BH FDR <0.05) for Cortex-EUR

219 (Supplementary Table 8). Of these, 632 (42\%) were an ieQTL in neurons, likely because this is

220 the most prevalent cell type. The majority of the ieQTLs $(90.2 \%)$ were uniquely mapped to one

221 cell type (Figure 4C). Although we observed a lower proportion of ieQTLs in cerebellum (126;

$2221.5 \%$, Supplementary Figure 17, Supplementary Table 8), this is likely a power issue due to

223 the smaller sample size. While we observed the most ieQTLs for neurons in cortex, the majority

$224(\mathrm{n}=106 ; 84 \%)$ of ieQTLs in cerebellum were mediated by astrocytes and macrophages.

226 We compared the allelic direction of the identified ieQTLs for each cell type with matching cell

227 types from a single nucleus RNA-seq (snRNA-seq) dataset (ROSMAP cohort, n=39;

228 Supplementary Table 9) ${ }^{28}$. When filtering on cell type mediated eQTLs by Decon-QTL

229 (FDR <0.05), we observed a high average concordance in allelic direction for both the eQTL

230 main effect (68\%), as well as the direction of the interaction (68\%; Supplementary Figure

231 18B). 106 of the cortex cis-ieQTLs were also significant $(\mathrm{BH}$ FDR $<0.05)$ in the snRNA-seq

232 datasets (63 in excitatory neurons and 43 in oligodendrocytes). Of these, 13 excitatory neuron

233 and 21 oligodendrocyte ieQTLs were cell type mediated by the corresponding cell type in bulk 
234 with 100\% allelic concordance (Decon-QTL; BH FDR <0.05; Supplementary Figure 18D). The

235 ieQTLs replicating in oligodendrocytes included STMN4, NKAIN1, and FAM221A (Figure 4D

236 and E and Supplementary Figure 19A-C), which have previously been identified as

237 oligodendrocyte specific ${ }^{29}$. Additionally, this set of ieQTLs included AMPD3 (rs11042811) and

238 CD82 (rs2303865), genes involved in the white matter microstructure ${ }^{30}$, suggesting a role for

239 oligodendrocytes in this pathway. The ieQTLs replicating in excitatory neurons included

240 SLC25A27 (alias UCP4; Figure 4F and Supplementary Figure 19D), a gene principally

241 expressed in neurons ${ }^{31}$ that modulates neuronal metabolism ${ }^{32}$. The eQTL SNP for this gene,

242 rs2270450, is in high $\operatorname{LD}\left(\mathrm{r}^{2}=0.71\right)$ with a variant previously associated with schizophrenia ${ }^{33}$.

243 Previous work has suggested a possible role of this gene in Parkinson's disease ${ }^{34,35}$. These results

244 suggest that the decomposition of eQTLs to their relevant cell types in MetaBrain yields

245 additional valuable information about the underlying biological mechanisms of genes and cell

246 types of interest for genes associated with disease.

\section{Shared genetic effects between Cortex-EUR cis-eQTLs and brain-related traits}

249 As one application of the MetaBrain resource, we linked cis-eQTLs to variants associated with

250 brain-related traits and diseases. For this, we first evaluated linkage disequilibrium (LD) between

251 the Cortex-EUR cis-eQTL SNPs with the strongest association signals and index variants

252 identified in 1,057 GWASs of brain-related traits (Supplementary Note, Supplementary Table

253 10). We observed that $10 \%$ of brain-related trait SNPs for $242 \mathrm{eQTL}$ genes were in LD with cis-

254 eQTL SNPs $\left(r^{2}>0.8\right)$. This percentage marginally increased to $12 \%$ when secondary, tertiary and

255 quaternary eQTL SNPs were included, indicating that the majority of LD overlap is driven by 
256 primary eQTL effects: primary eQTLs were 3.3-fold more likely to be in LD with a GWAS SNP

257 (Fisher exact test $\mathrm{p}$-value $=6.2 \times 10^{-16} ;$ Supplementary Note) .

259 To more formally test for overlap between GWAS and cis-eQTL signals, we conducted

260 Mendelian randomization (MR) to test for a causal effect between gene expression and 31

261 neurological traits using cis-eQTLs as instruments (Supplementary Table 11). We computed a

262 Wald ratio for each eQTL instrument, from which 1,192 Wald ratios out of 268,030 tested in

263 total passed a suggestive $\mathrm{p}$-value threshold $\left(\mathrm{p}<5 \times 10^{-5} ;\right.$ Supplementary Table 12). 120 of the cis-

264 eQTL instruments from these suggestive findings were also cell type ieQTLs. We further

265 prioritized our list of genes with evidence of Wald ratio effects by determining genetic

266 colocalization between GWAS and cis-eQTL signals using coloc ${ }^{36}$. There were 159 significant

267 Wald ratios that passed a strict Bonferroni correction $\left(\mathrm{p}<1.87 \times 10^{-7}\right)$ where the GWAS SNP and

268 eQTL colocalized (PP4>0.7; Figure 5A; Supplementary Figure 20). 69 of these prioritized

269 findings were associated with neurological and neuropsychiatric disease risk (Table 1). Three

270 examples where MR and colocalization pointed to likely causal GWAS genes are reported

271 below, for others, see Supplementary Note, Supplementary Tables 11-16 and

272 Supplementary Figures 21 and 22.

273

274 MR comparison between blood and brain eQTL datasets

275 MR analysis for multiple sclerosis (MS) ${ }^{37}$ identified 102 instruments in 83 genes that passed the

276 Bonferroni-adjusted p-value threshold (Supplementary Table 12). 20 of these findings passed

277 colocalization (Table 1; Figure 5B). This included 11 genes for which MR suggested that

278 increased gene expression and 9 genes where decreased gene expression may confer MS risk. 
279 Systematic comparison of the Wald ratio estimates for MS of 5,919 shared cis-eQTL genes

280 between Cortex-EUR and eQTLGen (where the same gene was instrumented but could be with

281 different SNPs) ${ }^{17}$ showed opposite directions of effect for 2,291 (38.7\%) genes (Supplementary

282 Figure 23, Supplementary Table 14). Agreement improved when the same SNP instrument

283 was compared between studies, but discordance still remained high with 1,891 (26\%) out of

2847,274 MetaBrain Wald ratios showing opposite directionality to eQTLGen (Supplementary

285 Table 15). The notable discordance in the directionality of the blood and brain eQTLs

286 underscore the importance of tissue-specific differences when interpreting transcriptomics data.

288 Of the 135 genes with MR findings in Cortex-EUR for MS, there were 28 genes without a 289 significant eQTLGen instrument, including 3 genes (SLC12A5, CCDC155 and MYNN) for which 290 we found both MR significance and colocalization in MetaBrain (Supplementary Note;

291 Supplementary Table 16. Comparing blood and brain gene expression levels for these genes in

292 GTEx, SLC12A5 had almost no expression in blood, while expression was comparable between

293 tissues for CCDC155 and MYNN (Supplementary Note, Supplementary Figure 24). The

294 discrepancy in MR findings observed between Cortex-EUR and eQTLGen suggest tissue-

295 dependent genetic effects for MS.

297 MR and colocalization analysis links multiple sclerosis GWAS loci to cell type specific 298 eQTLs for CYP24A1 and CLECL1

299 Two MS genes, CYP24A1 and CLECL1, showed cell type specific cis-eQTLs (Figure 5C and

300 D). Another gene that was previously suggested to be neuron specific ${ }^{38}, S L C 12 A 5$, did not show

301 a significant ieQTL in our data. Our analysis used rs2259735 as the Cortex-EUR eQTL 
302 instrument variable and suggested that higher expression of CYP24A1 is associated with

303 increased MS risk (MR Wald ratio=0.13, $\mathrm{p}=1.7 \times 10^{-9}$ ). We also observed colocalization of the

304 cis-eQTL and the MS GWAS signal at this region (coloc PP4=0.99), suggesting the same

305 underlying genetic signal. Furthermore, ieQTL analysis showed increasing expression of

306 CYP24A1 with increasing neuronal proportions for the MS risk allele rs2248137 (interaction

307 beta $=2.85$; interaction FDR $=1 \times 10^{-308}$; Figure 5C). Rs2248137 has previously been associated

308 with $\mathrm{MS}^{39}$ and is in strong LD with SNP rs2259735 $\left(\mathrm{r}^{2}=0.9\right)$. CYP24Al is a mitochondrial

309 cytochrome $\mathrm{P} 450$ hydroxylase that catalyzes the inactivation of 1,25 -dihydroxyvitamin $\mathrm{D}_{3}$

310 (calcitriol), the active form of vitamin $\mathrm{D}^{40}$. Loss of function mutations in CYP24A1 increase

311 serum calcitriol and cause hereditary vitamin D-mediated PTH-independent hypercalcemia ${ }^{41,42}$.

312 In the brain, vitamin D plays vital functions in regulating calcium-mediated neuronal

313 excitotoxicity, reducing oxidative stress and regulating synaptic activity ${ }^{43}$. Epidemiological

314 studies have proposed vitamin D deficiency as a risk factor for MS ${ }^{44,45}$, which has recently been

315 validated through $\mathrm{MR}^{46-48}$. Our findings are consistent with a previous report of a shared MS

316 GWAS signal and CYP24A1 cis-eQTL signal with frontal cortex but not white matter, using a

317 brain eQTL dataset derived from expression microarrays to confirm the findings in the same

318 direction of effect ${ }^{49}$.

320 As another MS signal that passed MR and colocalization, decreased expression of CLECL1 was

321 associated with increased MS risk (MR Wald ratio=-0.16, $\mathrm{p}=1.58 \times 10^{-9}$, coloc PP4>0.92). The

322 ieQTL analysis indicated that the rs7306304 allele increased expression of CLECL1 with

323 increasing macrophage proportion (interaction beta=-3.65; interaction $\mathrm{FDR}=1 \times 10^{-308}$, Figure

324 5D), confirming a previous finding of a microglia cell-type specific cis-eQTL for CLECL1 at this 
325 MS risk locus ${ }^{39}$. Rs7306304 is in strong LD with the MS lead SNP, rs7977720 $\left(\mathrm{r}^{2}=0.84\right)^{39}$.

326 CLECL1 encodes a C-type lectin-like transmembrane protein highly expressed in dendritic and B

327 cells that has been proposed to modulate immune response ${ }^{50}$. CLECL1 was previously found to

328 be lowly expressed in cortical bulk RNA-seq data, while having a 20-fold higher expression in a

329 purified microglia dataset ${ }^{39}$, suggesting that decreased CLECL1 expression increases MS

330 susceptibility through microglia-mediated dysregulation of immune processes in the brain.

\section{MetaBrain allows for the identification of trans-eQTLs}

333 Trans-eQTL analysis can identify the downstream transcriptional consequences of disease

334 associated variants. However, we have previously observed in blood that trans-eQTL effect-sizes

335 are usually small. Here we studied whether this applies to brain as well. In order to maximize

336 sample size and statistical power, we performed a trans-eQTL analysis in 3,111 unique

337 individuals. We reduced the number of tests performed by limiting this analysis to 130,968

338 unique genetic variants: these include variants that have been previously found to be associated

339 with diseases and complex traits through GWAS and variants that were primary, secondary,

340 tertiary or quaternary lead cis-eQTL SNPs from any of the aforementioned discovery datasets.

342 We identified 3,940 trans-eQTLs (FDR<0.05), of which 2,589 (66\%) were significant after

343 removing trans-eQTLs for which the gene that partially map within 5Mb of the trans-eQTL SNP

344 (Supplementary Note; Figure 6A; Supplementary Table 17). These 2,589 eQTLs reflect 373

345 unique SNPs, and 1,263 unique genes. 222 (60\%) of the trans-eQTL SNPs were a cis-eQTL

346 SNP, of which 42 (19\%) were a cis-eQTL index SNP in Cortex-EUR, and $22(10 \%)$ in tissues 
347 other than cortex. This suggests that trans-eQTLs can also be observed for cis-eQTLs index

348 SNPs identified in other tissues (Supplementary Table 17).

$3501,060(83 \%)$ of the observed trans-eQTL genes were affected by 3 variants at $7 \mathrm{p} 21.3$

351 (rs11974335, rs10950398 and rs1990622, LD r $^{2}>0.95$; Figure 6A and B; Supplementary Table

352 17). This locus is associated with several brain-related traits, including frontotemporal lobar

353 degeneration ${ }^{51}$ and major depressive $\operatorname{disorder}^{52}$ (Supplementary Table 17). The trans-eQTL

354 SNP rs1990622 in this locus is the lead GWAS SNP for the TDP-43 subtype of frontotemporal

355 lobar degeneration (FTLD-TDP) ${ }^{53}$, just downstream of TMEM106B. Matching previous

356 reports ${ }^{54,55}$, we observed that this locus was associated with predicted neuron proportions

357 (Supplementary Tables 18-20). Moreover, the predicted neuronal proportions were lower in

358 AD cases than controls (Supplementary Figure 25), which may explain why a large number of

359 trans-eQTLs signals at this region were most pronounced in the AMP-AD datasets and had

360 stronger effect sizes in AD samples (Supplementary Figure 26 and 27). We performed

361 functional enrichment on the trans-eQTL genes using g:Profiler ${ }^{56}$ and observed that upregulated

362 trans-eQTL genes were enriched for neuron related processes such as synaptic signaling

$363\left(\mathrm{p}=1.3 \times 10^{-28}\right)$ and nervous system development $\left(\mathrm{p}=2.9 \times 10^{-21}\right)$. Downregulated genes were

364 enriched for gliogenesis $\left(\mathrm{p}=1.6 \times 10^{-8}\right)$ and oligodendrocyte differentiation $\left(\mathrm{p}=3.1 \times 10^{-6}\right.$;

365 Supplementary Table 21). Surprisingly, 21 of these trans-eQTLs were also significant (BH

366 FDR $<0.05)$ in the snRNA-seq data of excitatory neurons with $100 \%$ allelic concordance

367 (Supplementary Figure 28; Supplementary Table 22), suggesting that some of these trans-

368 eQTLs might not be driven by differences in neuron proportions. A detailed description of this

369 locus can be found in the Supplementary Note. 
371 We observed trans-eQTLs from multiple independent genomic loci for 14 genes, suggesting

372 convergent trans-eQTL effects (Supplementary Table 17). The genes with these convergent

373 trans-eQTL effects were previously associated with immunological phenotypes (HBG2, PIWIL2,

374 and SVEP1), brain-related phenotypes (DAZAP2), immunological and brain-related phenotypes

375 (HMCES, KCNA5, MBTPS1, PRPF19, PTH2R and RFPL2) or other phenotypes (ANKRD2,

376 PEX12, PROM1 and ZNF727).

378 Encouragingly, some of these convergent trans-eQTLs have already been previously identified

379 in blood. For example, two independent variants (rs1427407 on 2p16.1 and rs4895441 on

380 6q23.3) affected hemoglobin subunit gamma-2 (HBG2) on 11p15.4 in trans (Figure 6C). These

381 variants have previously been associated with fetal hemoglobin levels ${ }^{57-59}$ and various blood cell 382 counts.

384 We also observed converging effects that were not identified in blood. For instance, KCNA5

385 (12p13.32) was affected by variants from three independent loci at 2p23.3 (rs930263), 4p15.32

386 (rs2702575 and rs2604551) and 7p21.3 (rs10950398 and rs11974335) as described in Figure

387 6C; Supplementary Table 17. KCNA5 encodes the potassium voltage-gated channel protein

388 Kv1.5. Potassium voltage-gated channels regulate neuron excitability among other functions, and

389 blockers for these channels have been suggested as a therapeutic target for multiple sclerosis

390 patients $^{60}$. Furthermore, KCNA5 has previously been associated with cardiovascular disease ${ }^{61}$,

391 and has been suggested to modulate macrophage and microglia function ${ }^{62}$. Three cis-eQTLs $^{2}$

392 were associated with rs930263, including $A D G R F 3, D R C 1$, and a secondary eQTL on $H A D H B$. 
393 rs930263 was previously associated with sleep dependent LDL levels ${ }^{63}$ and several blood

394 metabolite levels ${ }^{64-67}$. The 4p15.32 locus was previously associated with insomnia and adult

395 height $^{68}$ and the 7 p21.3 locus with depression and blood protein levels. These results thus

396 suggest that several sleep related variants affect potassium voltage-gated regulation of neuron

397 excitability.

399 This is the first report of trans-eQTLs in the brain cortex for many of the variants identified, and

400 our results indicate that many of these signals are brain-specific. We observed the trans-eQTL

401 effect-sizes in brain are usually small, similar to what we previously observed in blood,

402 emphasizing the importance of increasing the sample-size of brain eQTL studies.

404 Brain co-regulation networks improve GWAS interpretation

405 We generated brain-region specific co-regulation networks based on the RNA-seq data from

406 8,544 samples (Supplementary Note, Supplementary Figures 29-30). We previously have

407 done this for a heterogenous set of RNA-seq samples spanning across all available tissue types

408 and cell lines $(n=31,499)^{69,70}$, which showed that such a co-regulation network can be

409 informative for interpreting GWAS studies ${ }^{69}$ and helpful in the identification of new genes that

410 cause rare diseases ${ }^{70}$.

412 We applied a new approach ('Downstreamer', in preparation, see Supplementary Note) that

413 improves upon DEPICT, our previously published post-GWAS pathway analysis method ${ }^{69}$.

414 Downstreamer can systematically determine which genes are preferentially co-regulated with

415 genes that reside within GWAS loci. It does not use a significance threshold for a GWAS, but 
416 instead uses all SNP information. In addition, Downstreamer accounts for LD and uses rigorous

417 permutation testing to determine significance levels and control for Type I errors.

419 We applied Downstreamer to schizophrenia $(\mathrm{SCZ})^{71}, \mathrm{PD}^{72}, \mathrm{MS}^{37}, \mathrm{AD}^{73}$ and ALS GWAS

420 summary statistics (Supplementary Table 23-27), using three different brain-derived co-

421 regulation networks: one based on all 8,544 brain samples, one limited to 6,527 cortex samples

422 and one limited to 715 cerebellum samples. We observed that there were multiple sets of genes

423 that showed strong co-regulation with genes inside the GWAS loci for these diseases. For MS

424 and $\mathrm{AD}$, these were mostly immune genes, whereas for PD, ALS and SCZ these were genes that

425 are specifically expressed in brain (Supplementary Table 23-27).

427 For ALS, we applied Downstreamer to summary statistics from a recent meta-analysis in

428 individuals from European ancestry (Supplementary Table 28), and a trans-ethnic meta-

429 analysis including European and Asian individuals (EUR+ASN; Supplementary Table 23; van

430 Rheenen et al., manuscript in preparation). To look for contributions of non-neurological cell

431 types and tissues, we first used the previously published heterogenous network ${ }^{70}$ that comprises $^{2}$

432 many different tissues and cell types, but did not identify genes that were significantly enriched

433 for co-regulation with genes inside ALS loci. However, when we applied our method to the

434 different brain co-regulation networks, we identified a set of 27 unique co-regulated genes

435 (EUR+ASN summary statistics; Figure 7A; Supplementary Table 23), depending on the type

436 of brain co-regulation network used. HUWE1 was shared between the brain and cortex co-

437 regulation network analysis, while $U B R 4$ was shared between the cortex and cerebellum

438 analysis. $U B R 4$ is a ubiquitin ligase protein expressed throughout the body. A private $U B R 4$ 
mutation, segregated with episodic ataxia in a large three-generation Irish family, implicates its

440 role in muscle coordination ${ }^{74} . U B R 4$ interacts with the $\mathrm{Ca}^{2+}$ binding protein, calmodulin and $\mathrm{Ca}^{2+}$

441 dysregulation has been linked to proteins encoded by ALS disease genes and motor neuron

442 vulnerability ${ }^{75}$. We observed in the Downstreamer findings that many of these prioritized genes

443 are co-regulated with each other (Figure 7B), and using our recently developed clinical symptom

444 prediction algorithm ${ }^{70}$, there was an enrichment of genes implicated in causing gait disturbances

445 (Figure 7C). These genes are associated with ALS (highlighted in blue), brain-related disorders

446 (including DNAJC5, HTT, HUWE1, TSC1 and YEATS2) or muscle-related disorders (including

$447 K M T 2 B)$. While various loci have been identified for both familial and sporadic forms of ALS,

448 the function of the positional candidate genes within these loci is still unclear. Our

449 Downstreamer analysis identified genes that show strong coregulation with positional candidate

450 genes inside ALS loci, suggesting that these positional candidates must have a shared biological

451 function.

453 For MS, the heterogeneous network, including many blood and immune cell type samples,

454 identified 257 unique genes that showed significantly enriched co-regulation with genes inside

455 MS loci (Figure 7D; Supplementary Table 27), and many were immune genes, which is also

456 expected for this disease. However, when we applied the brain co-regulation networks, we

457 identified a much smaller set of genes, and these genes showed strong enrichment for genes

458 involved in the neurotrophin signaling pathway (Figure $\mathbf{7 E}$ and $\mathbf{F}$ ). Neurotrophins are

459 polypeptides secreted by immunological cell types. In the brain, neurotrophin concentrations are

460 important to promote the survival and proliferation of neurons as well as synaptic transmission.

461 In MS patients, neurotrophin reactivity is higher in MS plaques, whereby neurotrophins are 
462 released by peripheral immune cells directly to the inflammatory lesions, suggesting a protective

463 role of this signaling process ${ }^{76,77}$. Neutrophins are also released by glial cells in the brain,

464 including microglia and astrocytes, and their role in stimulating neuronal growth and survival

465 could also contribute to an overall neuroprotective effect ${ }^{78}$. In the heterogeneous network, we

466 observed high expression for these genes in immune-related tissues (Supplementary Figure

467 31A), supporting the "outside-in hypothesis" that the immune system may be a potential trigger

468 for $\mathrm{MS}^{37,79}$. The brain specific network showed high expression in spinal cord and cerebellum

469 but lower expression in cortex samples (Supplementary Figure 31B), which could be

470 highlighting the specific biological processes taking place in these CNS regions that lead to

471 disease. For example, the cerebellum is responsible for muscle coordination and ataxia occurs in

472 approximately $80 \%$ of MS patients with symptoms ${ }^{80}$. We speculate that both dysregulation of the

473 immune system and dysregulation of certain neurological processes is a prerequisite for

474 developing MS.

475

\section{Discussion}

477 We here describe an integrated analysis of the effects of genetic variation on gene expression

478 levels in brain in over 3,000 unique individuals. This sample size yielded sufficient statistical

479 power to identify robust cis-eQTLs and to our knowledge for the first-time brain trans-eQTLs

480 that emanate from SNPs previously linked to neurodegenerative or psychiatric diseases.

482 We compared cis-eQTLs in MetaBrain to cis-eQTLs in eQTLGen from a set of 31,684 blood

483 samples. We observe a large proportion of shared cis-eQTLs between brain and blood, most of

484 which have the same allelic direction of effect. Our analysis also permitted us to identify cis- 
485 eQTL effects that are independent of the primary cis-eQTLs. Some of these independent effects

486 reflect SNPs that are also the index variants for several neurological and psychiatric disorders,

487 making them particularly interesting for subsequent follow-up. Recent observations have

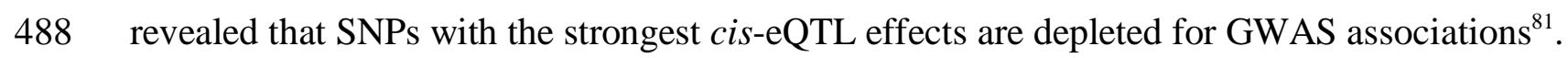

489 Thus, secondary, tertiary or quaternary cis-eQTL SNPs could potentially be even more

490 interesting to follow-up than certain primary cis-eQTL SNPs to link association signals to

491 function.

492

493 We studied different regions in the brain, permitting us to identify brain-region specific eQTLs.

494 For this, to exclude spurious differences that may arise from different cell type proportions

495 across brain regions, we first inferred cell type percentages for the major brain cell types. We

496 then applied an eQTL interaction model (i.e., using the cell type percentage $\mathrm{x}$ genotype as

497 interaction term), permitting us to identify 1,515 cis-eQTLs that show cell type specificity. Most

498 of these cell type dependent effects were observed for oligodendrocytes and neurons, the two

499 most common cell types in the brain for which statistical power to observe such effects was the

500 strongest. Still, we could identify 461 cell type dependent eQTLs also for macrophages,

501 endothelial cells, or astrocytes. While we found strong concordance with immunohistochemistry

502 results, our findings are largely based on a deconvolution approach, which in future studies will

503 benefit from validation in purified cell types, e.g. using population-based single-cell RNA-seq

504 datasets as they are now becoming available ${ }^{82,83}$. Such single-cell eQTL studies can gain

505 substantial statistical power by limiting analyses to the large set of primary, secondary, tertiary

506 and quaternary cis-eQTLs our study reveals for bulk brain samples. 
508 To our knowledge, this is the best powered Mendelian randomization and colocalization analysis

509 using brain cis-eQTLs as instruments for bipolar disease, epilepsy, frontotemporal dementia,

510 multiple sclerosis, cognitive function and years of schooling GWAS outcomes. Interestingly,

511 also for schizophrenia three signals for CILP2, MAU2 and TM6SF2 met our criteria that had not

512 been reported in a recent psychiatric genomics consortium study ${ }^{84}$, further emphasizing the value

513 of our well-harmonized, large eQTL data set in the tissue type of interest (Supplementary

514 Note). Our results also identify increased $C Y P 24 A 1$ expression as associated with multiple

515 sclerosis risk and propose neurons as the most susceptible cell type to CYP24A1 expression

516 changes and likely active vitamin D levels. The potentially novel role of $C Y P 24 A 1$ in brain could

517 play an important role in MS etiology, as may lowered expression of CLECL1 in microglia.

519 The 2,589 identified trans-eQTLs allowed us to gain insights into downstream molecular

520 consequences of several disease-associated genetic variants. Our trans-eQTL analysis focused on

521 a single brain region and SNPs with a known interpretation (i.e. trait-associated variants and cis-

522 eQTL SNPs). We therefore expect that a genome-wide approach will identify many more trans-

523 eQTLs. 2,218 of the trans-eQTLs were located in a 7p21.3 locus and the genes were strongly

524 correlated with neuron proportions, indicating that cell type proportions can heavily impact

525 trans-eQTL identification. However, 21 of these trans-eQTLs replicated in snRNA-seq data,

526 suggesting that some of these trans-eQTLs may also exist in single cells. Excluding the 7p21.3

527 locus, we identified 371 trans-eQTLs located elsewhere in the genome, which are less likely due

528 to neuron proportion differences. For several neurological and psychiatric conditions, our

529 analyses indicate pathways that may help to elucidate disease causes and putative intervention

530 points for future therapies. 
532 We used the brain-specific co-regulation networks to study several brain-related GWAS studies,

533 with the aim to prioritize genes that show significantly enriched co-regulation with genes inside

534 the associated GWAS loci. For ALS this revealed a limited, but significant set of genes which do

535 not map within associated ALS loci, but that link genes within multiple ALS loci. Follow-up

536 research on these prioritized genes might therefore help to better understand the poorly

537 understood causal pathways that cause ALS. While it is tempting to speculate that these

538 prioritized genes might represent genes that could serve as potential targets for pharmaceutical

539 intervention, follow-up research is needed in order to establish whether these genes play a

540 relevant role in ALS.

542 Our study had several limitations. For instance, we performed single tissue eQTL analyses that

543 were limited to a single RNA-seq sample per individual, excluding many RNA-seq samples from

544 the analysis. A joint analysis across tissues, including multiple RNA-seq samples per individual

545 using for example random effects models would further improve power ${ }^{85,86}$, which would be

546 especially useful for the future identification of trans-eQTLs. Additionally, LD overlap analysis,

547 Mendelian randomization and colocalization are sensitive to many factors, including eQTL and

548 GWAS study sample size, effect size, variant density, LD structure and imputation quality.

549 Differences between study designs may consequently influence the results of such analyses. For

550 example, our colocalization and LD overlap analysis did not include the MAPT gene for

551 Alzheimer's disease. The effect sizes of the cis-eQTLs for this gene were limited in our study,

552 since our alignment strategy could not account for the different long-range haplotypes in this

553 locus causing the $\mathrm{H} 1 / \mathrm{H} 2$ haplotype separating SNP rs8070723 to have a p-value of 0.2 
554 (Supplementary Note). We note that this might be an issue for other genes as well. Future

555 studies using graph-based alignment tools or long read sequencing methods would be required to

556 ultimately determine the true effects on such genes. Our approach combined Mendelian

557 randomization and colocalization, as it is possible for the cis-eQTL instrument to coincidentally

558 share association with the GWAS trait due to surrounding LD patterns in the genomic region.

559 We opted to perform single SNP MR because other approaches, such as inverse variance

560 weighted $^{87}$ (IVW) MR, pool the estimates across many SNP instruments, which for many genes

561 were not available. Potentially, methods such as IVW could be applied to our dataset in the

562 future when genome-wide trans-eQTL analysis would identify many more independent

563 instruments per gene. However, MR analyses using QTLs could be susceptible to confounding

564 because of horizontal pleiotropy ${ }^{88}$, where a single gene is affected by multiple indirect effects,

565 which is likely to be exacerbated by including trans-eQTLs. Our colocalization analysis used a

566 more lenient posterior probability (PP4) threshold of $>0.7$, which we selected because we

567 performed colocalization only in loci having a significant MR signal, limiting potential false

568 positives. However, our colocalization approach assumed the presence of a single association in

569 each locus, which might not be optimal for cis-eQTL loci harboring multiple independent

570 variants, such as for the TREM2 gene (Supplementary Note). Consequently, our approach may

571 have not detected colocalizing signals in some loci. Recently, colocalization methods were

572 published $^{89}$ that do not have this assumption, and consequently may improve future

573 colocalization results.

575 With the numbers of GWAS loci for brain-related traits and diseases steadily climbing, we 576 expect that our resource will prove itself as a highly valuable toolkit for post-GWAS brain 
577 research and beyond. Among others, we demonstrate how our dataset can be utilized to

578 disambiguate GWAS loci, point to causal pathways and prioritize targets for drug discovery. To

579 our knowledge, this is the largest non-blood eQTL analysis ever conducted, providing insights

580 into the functional consequences of many disease associated variants. We expect that through

581 future integration with single-cell eQTL studies that have higher resolution but lower power, our

582 results will help to pinpoint transcriptional effects in specific brain cell types for many disease-

583 associated genetic variants. 
585 Methods

\section{Dataset collection and description}

587 We collected human brain bulk RNA-seq datasets from different resources. Briefly, we collected 588 previously published samples from the AMP-AD consortium ${ }^{6}$ (AMP-AD MAYO ${ }^{6}$, ROSMAP $^{6}$ $589{\left.\text { and } \text { MSBB }^{6}\right) \text {, Braineac }}^{7}$, the PsychENCODE consortium ${ }^{8}\left(\right.$ Bipseq $^{4}$, BrainGVEX $^{4}, \mathrm{CMC}^{9}$, GVEX, $^{2}$ 590 and UCLA_ASD ${ }^{4}$ ) from Synapse.org using the Python package synapseclient ${ }^{90}$. The NABEC and 591 GTEx datasets were retrieved from NCBI dbGaP, and TargetALS data was provided directly by 592 the investigators. For an overview of the number of samples per dataset, see Supplementary 593 Table 1.

595 Additionally, we collected public brain bulk RNA-seq samples from the European Nucleotide

596 Archive (ENA; Supplementary Table 28). To select only the brain samples, we first

597 downloaded the SkyMap database ${ }^{91}$, which provides readily mapped read counts and sample

598 annotations. We performed rigorous quality control on this dataset, and selected ENA, excluding

599 for example brain cell lines, brain cancer samples, and samples with RNA spike ins (See

600 Supplementary Note for more details on this method, Supplementary Figure 1), resulting in

6011,759 samples, and 9,363 samples when combined with the previously published datasets

602 (Supplementary Table 1).

603

604 RNA-seq data

605 RNAseq data was processed using a pipeline built with molgenis-compute ${ }^{92}$. FASTQ files were 606 aligned against the GENCODE ${ }^{93}$ v32 primary assembly with $\operatorname{STAR}^{94}$ (version 2.6.1c), while 607 excluding patch sequences (see Supplementary Note) with parameter settings: 
608 outFilterMultimapNmax = 1, twopassMode Basic, and outFilterMismatchNmax = 8 for paired-

609 end sequences, outFilterMismatchNmax $=4$ for single-end sequences. Gene quantification was

610 performed by STAR, similar to gene quantification using HTSeq ${ }^{95}$ with default settings. The

611 gene counts were then $\mathrm{TMM}^{96}$ normalized per cohort using edge $\mathrm{R}^{97}$ (version 3.20.9) with $\mathrm{R}^{98}$

612 (version 3.5.1).

613

614 To measure FASTQ and alignment quality we used FastQC ${ }^{99}$ version 0.11 .3 ), STAR metrics, and

615 Picard Tools ${ }^{100}$ (version 2.18.26) metrics (MultipleMetrics, and RNAseqMetrics). Samples were

616 filtered out if aligned reads had $<10 \%$ coding bases (Supplementary Figure 3A), $<60 \%$ reads

617 aligned (Supplementary Figure 3B), or <60\% unique mapping. 117 of the RNA-seq samples

618 did not pass this filter, mostly from $\mathrm{GTEx}^{97}$. The other quality measurements were visually

619 inspected but contained no outliers.

621 RNA-sequencing library preparation, and other technical factors can greatly influence the ability

622 to quantify of gene expression. Therefore, for a given sample such factors often influence the

623 total variation. For example, such issues can be caused by problems during RNA-seq library

624 preparation that led to an increased number of available transcripts to quantify, or conversely, a

625 lack of variation in quantified transcripts (compared to other samples in the dataset). We

626 therefore opted to identify RNA-seq outliers that were not explained by poor RNA-seq alignment

627 metrics. For this purpose, we performed PCA on the RNA data prior to normalization: we

628 reasoned that the first two components capture excess or depletion of variation caused by

629 technical problems. We identified 20 samples that were outliers in the PCA plot of the RNA-seq

630 data, where PC1 was more than 4 standard deviations from the mean (Supplementary Figure 
631 3A). Twenty outlier samples were removed and the principal components were recalculated

632 (Supplementary figure 3B). We detected and removed 45 additional outlier samples. We

633 confirmed no additional outlier samples in the third iteration and principal component

634 calculation, (Supplementary Figure 3C) and 8,868 samples were taken through additional QC.

636 We next removed genes with no variation and then log2-transformated, quantile normalized and

637 Z-score transformed the RNA-seq counts per sample. PCA on the normalized expression data

638 showed that datasets strongly cluster together (Supplementary Figure 4A), likely due to dataset

639 specific technical differences (e.g., single-end versus paired-end sequencing). To correct for this,

640 the normalized expression data was correlated against 77 covariates from different QC tools

641 (FastQC ${ }^{99}, \mathrm{STAR}^{94}$, and Picard Tools ${ }^{100}$ ), such as percent protein coding, GC content, and 5'

642 prime/3' prime bias. The top 20 correlated technical covariates (\% coding bases, $\%$ mRNA bases,

$643 \%$ intronic bases, median 3' prime bias, \% usable bases, \% intergenic bases, \% UTR bases, \%

644 reads aligned in pairs, average mapped read length, average input read length, number of

645 uniquely mapped reads, \% reads with improper pairs, number of reads improper pairs, total

646 sequences, total reads, \% chimeras, number of HQ aligned reads, number of reads aligned, HQ

647 aligned Q20 bases, HQ aligned bases) were regressed out of the expression data using a linear

648 model. After covariate correction, clustering of datasets in PC1 and PC2 were no longer present

649 (Supplementary Figure 4B).

651 Our collection of RNA-seq samples consisted of 36 different tissue labels, many of which were

652 represented by only a few samples. Therefore, we next defined major brain regions present in our

653 dataset, including samples from amygdala, basal ganglia, cerebellum, cortex, hippocampus and 
654 spinal cord. We noted that some samples (especially from ENA) were not annotated with a

655 specific major brain region. To resolve this, we performed PCA over the sample correlation

656 matrix and then performed k-nearest neighbors on the first two PCs $(k=7)$ to classify samples to

657 the major brain regions. Using this approach, we defined a set of 86 amygdala, 574 basal ganglia,

658723 cerebellum, 6,601 cortex, 206 hippocampus, 252 hypothalamus and 285 spinal cord samples

659 (Supplementary Table 1, Figure 2A).

660

661 Genotype data and definition of eQTL datasets

662 The genotype data for the included datasets was generated using different platforms, including

663 genotypes called from whole genome sequencing (WGS; AMP-AD, TargetALS ${ }^{12}$, GTEx $^{3}$ ),

664 genotyping arrays $\left(\mathrm{NABEC}^{11}\right.$, Braineac $\left.^{7}\right)$, and haplotype reference consortium $(\mathrm{HRC})^{101}$ imputed

665 genotypes (PsychENCODE datasets), or were called from RNA-seq directly (ENA dataset; see

666 Supplementary Note). In total, 22 different genotyping datasets were available, reflecting 6,658

667 genotype samples (Supplementary Table 1).

668

669 We performed quality control on each dataset separately, using slightly different approaches per

670 platform. For the array-based datasets, we first matched genotypes using GenotypeHarmonizer ${ }^{102}$

671 using 1000 genomes phase 3 v5a (1kgp) as a reference, limited to variants having MAF $>1 \%$,

$672<95 \%$ missingness and Hardy-Weinberg equilibrium p-value <0.0001. Genotypes were then

673 imputed using HRC v1.1 as a reference on the Michigan imputation server ${ }^{103}$. In all HRC

674 imputed datasets, variants with imputation info score $<0.3$ were removed. For the WGS datasets,

675 we removed indels and poorly genotyped SNPs having VQSR tranche <99.0, genotype quality

$676<20$, inbreeding coefficient $<-0.3$ and $>5 \%$ missingness, setting genotype calls with allelic depth 
$677<10$ and allelic balance $<0.2$ or $>0.8$ as missing. WGS datasets were not imputed with HRC.

678 Considering the small size of some of the datasets, we decided to focus further analysis on

679 variants with MAF $>1 \%$ and Hardy-Weinberg p-value $>0.0001$.

681 In each dataset, we removed genetically similar individuals by removing individuals with pihat

$682>0.125$, as calculated with PLINK $2.0^{104}$. Additionally, we merged genotypes with those from

683 1kgp, pruned genotypes with --indep-pairwise 5050.2 in PLINK, and performed PCA on the

684 sample correlation matrix. We performed k-nearest neighbors $(k=7)$ on the first two PCs, using

685 the known ancestry labels in $1 \mathrm{kgp}$, to assign an ancestry to each genotyped sample. The majority

686 of included samples was of EUR descent: 5,138 samples had an EUR assignment, 805 samples

687 had an AFR assignment, and 573 samples were assigned to the other ethnicities (Supplementary

688 Table 1, Figure 2B).

690 For the purpose of eQTL analysis, we next assessed links between RNA-seq and genotype

691 samples and noted that some individuals had multiple RNA-seq samples (e.g. from multiple

692 brain regions) or multiple genotype samples (e.g. from different genotyping platforms). In total,

693 we were able to determine 7,644 links between RNA-seq samples and genotype samples

694 (Supplementary Table 1), reflecting 3,525 unique EUR individuals, 624 unique AFR

695 individuals and 510 unique individuals assigned to other ethnicities. We then grouped linked

696 RNA-seq samples based on ethnicity and tissue group to prevent possible biases on eQTL

697 results. For those individuals with multiple linked RNA-seq samples, we selected a sample at

698 random within these groups. Within each tissue and ethnicity group, we then selected unique

699 genotype samples across datasets in such a way to maximize sample size per genotype dataset. 
700 For the eQTL analysis per tissue, we only considered those datasets having more than 30 unique

701 linked samples available, and for which at least two independent datasets were available. Using

702 these criteria for sample and dataset selection, we were able to create 7 eQTL discovery datasets:

703 Basal ganglia-EUR ( $\mathrm{n}=208)$, Cerebellum-EUR $(\mathrm{n}=492)$, Cortex-EUR ( $\mathrm{n}=2,970)$, Cortex-AFR

704 (n=420), Hippocampus-EUR (n=168) and Spinal cord-EUR (n=108; Supplementary Table 1,

705 Figure 2C).

706

707 eQTL analysis

708 Our dataset consists of different tissues and ethnicities, and samples have been collected in

709 different institutes using different protocols. Consequently, combining these datasets to perform

710 eQTL analysis is complicated, due to possible biases each of these factors may introduce. To

711 resolve this issue, we opted to perform an eQTL meta-analysis within each of the defined eQTL

712 discovery datasets. To reduce the effect of possible gene expression outliers, we calculated

713 Spearman's rank correlation coefficients for each eQTL in each dataset separately, and then

714 meta-analyzed the resulting coefficients using a sample size weighted Z-score method, as

715 described previously ${ }^{14}$. While we acknowledge that this method may provide less statistical

716 power than the commonly used linear regression, we chose this method to provide conservative

717 effect estimates. To identify cis-eQTLs, we tested SNPs located within $1 \mathrm{Mb}$ of the transcription

718 start site, while for the identification of trans-eQTLs, we required this distance to be at least 5

$719 \mathrm{Mb}$. For both analyses, we selected variants having a MAF>1\%, and a Hardy-Weinberg p-value

$720>0.0001$. Using the GENCODE v32 annotation, we were able to quantify 58,243 genes, of which

72119,373 are protein coding. While non-coding genes have been implicated to be important for

722 brain function ${ }^{105}$, these genes generally have poor genomic and functional annotations, meaning 
723 that it is often unknown in which pathway they function, and that there is uncertainty about their

724 genomic sequence. We therefore focused our eQTL analyses on protein coding genes.

726 To correct for multiple testing, we reperformed the cis- and trans-eQTL analyses, while

727 permuting the sample labels 10 times. Using the permuted p-values, we created empirical null

728 distributions and determined a false discovery rate (FDR) as the proportion of unpermuted

729 observations over the permuted observations and considered associations with FDR $<0.05$ as

730 significant. To provide a more stringent FDR estimate for our cis-eQTL results, we limited FDR

731 estimation to the top associations per gene, as described previously ${ }^{14}$. We note that our FDR

732 estimate is evaluated on a genome-wide level, rather than per gene, and consequently FDR

733 estimates stabilize after a few permutations ${ }^{106}$.

735 Since cis-eQTL loci are known to often harbor multiple independent associations, we performed

736 an iterative conditional analysis, where for each iteration, we regressed the top association per

737 gene from the previous associations, and re-performed the cis-eQTL analysis until no additional

738 associations at FDR $<0.05$ could be identified.

740 Since a genome-wide trans-eQTL analysis would result in a large multiple testing burden

741 considering the billions of potential tests, we limited this analysis to a set of 130,968 variants

742 with a known interpretation. This set constituted of variants that were either previously

743 associated with traits, having a GWAS p-value $<5 \times 10^{-8}$ in the IEU OpenGWAS database ${ }^{107}$ and

744 EBI GWAS catalog ${ }^{108}$ on May $3^{\text {rd }}, 2020$, and additional neurological traits (see Supplementary

745 Table 17) or were showing an association with FDR $<0.05$ in any of our discovery cis-eQTL 
analyses (including secondary, tertiary and quandary associations identified in the iterative

747 conditional analysis). Cis-eQTLs in Cortex-EUR were highly concordant when replicated in

748 Cortex-AFR (Figure 3C). Consequently, to maximize the sample size and statistical power, we

749 meta-analyzed Cortex-EUR and Cortex-AFR datasets together. However, for the trans-eQTL

750 analysis we omitted ENA, to prevent bias by genotypes called from RNA-seq samples.

751 Additionally, For the trans-eQTL analysis, we did not correct the gene expression data for 10

752 PCs, since trans-eQTLs can be driven by cell proportion differences ${ }^{17}$, and many of the first 10

753 PCs in the MetaBrain dataset were correlated with estimated cell type proportions

754 (Supplementary Figure 32). To test for trans-eQTLs, we assessed those combinations of SNPs

755 and genes where the SNP-TSS distance was >5 Mb, or where gene and SNP were on different

756 chromosomes. We note that we did not evaluate eQTLs where the SNP-TSS distance was $>1 \mathrm{Mb}$

757 and $<5 \mathrm{Mb}$, which potentially excludes detection of long-range cis-eQTLs or short-range trans-

758 eQTLs. We expect however, that this excludes only a limited number of eQTLs, since we

759 observed that this distance was $<31 \mathrm{~Kb}$ for $50 \%$ of cis-eQTLs (Figure 3B), indicating most cis-

760 eQTLs are short-ranged. Additionally, we reasoned that the $>5 \mathrm{Mb}$ cutoff would prevent

761 identification of false-positive trans-eQTLs due to long-range LD.

762

763 Estimation of cell type proportions and identification of cell type mediated eQTLs

764 By leveraging cell type specific gene expression collected through scRNA-seq, a bulk tissue

765 sample can be modelled as a parts-based representation of the distinct cell types it consists of. In

766 such a model, the weights of each part (i.e. cell type proportions) can be determined by

767 deconvolution. In the deconvolution of the MetaBrain bulk expression data we used a single-cell

768 derived signature matrix including the five major cell types in the brain: neurons, 
769 oligodendrocytes, macrophages, endothelial cells and astrocytes. This signature matrix was

770 generated in the context of the CellMap project (Zhengyu Ouyang et al.; manuscript in

771 preparation). In short, we created pseudo-bulk expression profiles by extracting gene expression

772 values for specific cell types of interest from annotated single cell and single nuclei expression

773 matrices. Using differential expression analysis and applying several rounds of training and

774 testing, we selected 1,166 differentially expressed genes and calculated the average read counts

775 per cell type. We then filtered out genes that had no variation in expression, leaving a total of

776 1,132 genes. We extracted the corresponding TMM normalized gene counts of these signature

777 genes for all European cortex samples in MetaBrain. After correcting the counts for cohort

778 effects using OLS, but not for any other technical covariates, we applied $\log 2$ transformation on

779 both the signature matrix as well as the bulk gene count matrix. Subsequently we applied non-

780 negative least squares (NNLS) ${ }^{109}$ using SciPy (version 1.4.1) ${ }^{110}$ to model the bulk expression as a

781 parts-based representation of the single-nucleus derived signature matrix. First introduced by

782 Lawson and Hanson ${ }^{109}$, NNLS method is the basis of numerous deconvolution methods to date.

783 In short, NNLS attempt to find a non-negative weight (coefficient) for each of the cell types that,

784 when summed together, minimizes the least-squares distance to the observed gene counts.

785 Lastly, we transformed the resulting coefficients into cell type proportions by dividing them over

786 the sum of coefficients for each sample. The resulting cell proportions are then used to identify

787 cell type mediated eQTL effects. For this we applied Decon-eQTL ${ }^{22}$ (version 1.4; default

788 parameters) in order to systematically test for significant interaction between each cell type

789 proportion and genotype, while also controlling for the effect on expression of the other cell

790 types. The resulting p-values are then correct for multiple testing using the Benjamini-Hochberg

791 method on a per-cell-type basis. 


\section{Cell type specific ROSMAP single-nucleus datasets}

794 In order further confirm cell type specific eQTL effects, we used the ROSMAP single-nucleus

795 data, encompassing 80,660 single-nucleus transcriptomes from the prefrontal cortex of 48

796 individuals with varying degrees of Alzheimer's disease pathology ${ }^{111}$. We used Seurat version

$797 \quad 3.2 .2^{112}$ to analyze the data. First, we removed the genes that did not pass filtering as described 798 previously $^{111}$, leaving us with 16,866 genes and 70,634 cells for further analysis. After this, we

799 normalized the expression matrix on a per individual per cell type basis using sctransform ${ }^{113}$ and

800 visualized the normalized expression matrix using UMAP dimensionality reduction ${ }^{114}$. We

801 observed that cell types, as defined by Mathys et $a l^{115}$., for the majority cluster together

802 (Supplementary Figures 33 and 34). We then created expression matrices for each broad cell

803 type (excitatory neurons, oligodendrocytes, inhibitory neurons, astrocytes, oligodendrocyte

804 precursor cells, microglia, pericytes and endothelial cells) by calculating the average expression

805 per gene and per individual basis. We then used these cell-type datasets for eQTL mapping using

806 the same procedure as the bulk data. To correct for multiple testing, we confined the analysis to

807 only test for primary cis- and trans-eQTLs found in MetaBrain cortex, while also permuting the

808 sample labels 100 times. Lastly, we calculated the Spearman correlation between gene

809 expression levels and genotypes and their $95 \%$ confidence intervals ${ }^{116}$.

\section{Single SNP Mendelian Randomization analysis}

812 Mendelian Randomization (MR) was conducted between the Cortex-EUR eQTLs and 31

813 neurological traits (21 neurological disease outcomes, 2 quantitative traits and 8 brain volume

814 outcomes) (Supplementary Table 11). Cortex-EUR eQTLs at genome-wide significant 
$815\left(\mathrm{p}<5 \times 10^{-8}\right)$ were selected and then LD clumped to obtain independent SNPs to form our set of

816 instruments. LD clumping was carried out using the ld_clump() function in the ieugwasr

817 package ${ }^{117}$ on the default settings $\left(10,000 \mathrm{~Kb}\right.$ clumping window with $\mathrm{r}^{2}$ cut-off of $0.001 \mathrm{using}$

818 the 1000 Genomes EUR reference panel). SNP associations for each of the eQTL instruments

819 were then looked up in the outcome GWASs of interest. If the SNP could not be found in the

820 outcome GWAS using a direct lookup of the dbSNP rsid, then a proxy search was performed to

821 extract the next closest SNP available in terms of pairwise LD, providing minimum $\mathrm{r}^{2}$ threshold

822 of 0.8 with the instrument. Outcome GWAS lookup and proxy search was performed using the

823 associations() function in the ieugwasr package. To ensure correct orientation of effect alleles

824 between the eQTL instrument and outcome GWAS associations, the SNP effects were

825 harmonized using the harmonise_data() function in TwoSampleMR ${ }^{87}$. Action 2 was selected

826 which assumes that the alleles are forward stranded in the GWASs (i.e. no filtering or re-

827 orientation of alleles according to frequency was conducted on the palindromic SNPs). Single

828 SNP MR was then performed on the harmonized SNP summary statistics using the

829 mr_singlesnp() function in TwoSampleMR. Single SNP MR step computes a Wald ratio, which

830 estimates the change in risk for the outcome per unit change in gene expression, explained

831 through the effect allele of the instrumenting SNP. We reported all the MR findings that passed a

$832 \mathrm{p}$-value threshold of $5 \times 10^{-5}$, but note that the Bonferroni-corrected $\mathrm{p}=0.05$ threshold for multiple

833 testing correction is $\mathrm{p}=1.865 \times 10^{-7}$. We did not implement multi-SNP analysis (such as the

834 Inverse Variance Weighted method), because there are a small number of instrumenting SNPs

835 available per gene, which could result in unreliable pooled MR estimates for genes. 


\section{Colocalization}

838 Following the MR analysis, colocalization analysis was performed on the MR findings that

839 passed the suggestive threshold to determine if the eQTL and trait shared the same underlying

840 signal. We ran colocalization ${ }^{36}$ using both the default parameters $\left(\mathrm{p} 1=\mathrm{p} 2=10^{-4}\right.$ and $\left.\mathrm{p} 12=10^{-5}\right)$ and

841 parameters based on the number of SNPs in the region ( $\mathrm{p} 1=\mathrm{p} 2=1 /($ number of SNPs in the region)

842 and $\mathrm{p} 12=\mathrm{p} 1 / 10)$. We considered the two traits, eQTL and GWAS outcome to colocalize if either

843 of the two parameters yielded PP4>0.7. Additionally, colocalization was systematically analyzed

844 against one trait to compare to robustness of the Cortex-EUR eQTLs with existing cortex eQTL

845 data sets (see Supplementary Note). 


\section{URLS}

848 Picard: http://broadinstitute.github.io/picard/

849 dbGAP: https://dbgap.ncbi.nlm.nih.gov

850 European Nucleotide Archive: http://www.ebi.ac.uk/ena

851 ieugwasr package: https://mrcieu.github.io/ieugwasr/

852 TwoSampleMR: https://mrcieu.github.io/TwoSampleMR/

\section{Accessions}

855 TargetALS ${ }^{12}$ TargetALS data was pushed directly from the NY Genome center to our sftp

856 server.

857 CMC ${ }^{118}$ CMC data was downloaded from https://www.synapse.org/ using synapse client

858 (https://python-docs.synapse.org/build/html/index.html). Accession code: syn2759792

859 GTEx $^{86}$ GTEx was downloaded from SRA using fastq-dump of the SRA toolkit

860 (http://www.ncbi.nlm.nih.gov/Traces/sra/sra.cgi?cmd=show \&f=software \&m=software \&s=softw

861 are). Access has been requested and granted through dbGaP.

862 Braineac ${ }^{7}$ Braineac data has been pushed to our ftp server by Biogen.

863 AMP-AD ${ }^{5}$ AMP-AD data has been downloaded from synapse ${ }^{13}$. Accession code: syn2580853.

864 snRNA-seq was collected using Synapse accession code: syn18485175. IHC data:

865 https://github.com/ellispatrick/CortexCellDeconv/tree/master/CellTypeDeconvAnalysis/Data

866 ENA ${ }^{13}$ ENA data has been downloaded from the European Nucleotide Archive. The identifiers

867 of the 76 included studies and 2021 brain samples are listed in Supplementary Table 29. 
868 CMC_HBCC: CMC_HBCC data was downloaded from https://www.synapse.org/ using

869 synapse client (https://python-docs.synapse.org/build/html/index.html). Accession code:

$870 \quad$ syn 10623034

871 BrainSeq BrainSeq data was downloaded from https://www.synapse.org/ using synapse client

872 (https://python-docs.synapse.org/build/html/index.html). Accession code: syn12299750

873 UCLA_ASD UCLA_ASD data was downloaded from https://www.synapse.org/ using synapse

874 client (https://python-docs.synapse.org/build/html/index.html). Accession code: syn4587609

875 BrainGVEx BrainGVEx data was downloaded from https://www.synapse.org/ using synapse

876 client (https://python-docs.synapse.org/build/html/index.html). Accession code: syn4590909

877 BipSeq BipSeq data was downloaded from https://www.synapse.org/ using synapse client

878 (https://python-docs.synapse.org/build/html/index.html). Accession code: syn5844980

879 GTEx GTEx data was downloaded from dbgap. Accession code: phs000424.v7.p2

880 NABEC NABEC data was downloaded from dbgap. Accession code: phs001301.v1.p1

881 CellMap single-cell and single-nuclei RNA-seq datasets were downloaded from Gene

882 Expression Omnibus (GEO), BioProject, the European Genome-phenome Archive (EGA) and

883 the Allan Brain Atlas. Accession codes: GSE97930, GSE126836, GSE103723, GSE104276,

884 PRJNA544731, PRJNA434002, phs000424, phs001836. 


\section{Acknowledgements}

We thank the donors of the brain tissues underlying the RNA-seq data used for this study and their families for their willingness to donate samples for research. We would like to thank the Center for Information Technology of the University of Groningen for their support and for providing access to the Peregrine high-performance computing cluster, as well as the UMCG Genomics Coordination center, the UG Center for Information Technology and their sponsors BBMRI-NL and TarGet for storage and compute infrastructure. We would like to greatly thank all researchers involved with the following projects for making their data available for use. E.A.T., Y.H., C.-Y.C, E.E.M., M.I.Z. and H.R. are employed by Biogen. D.B. and T.R.G. are supported by funding from the UK Medical Research Council (MRC Integrative Epidemiology Unit at the University of Bristol, MC_UU_00011/4) and a sponsored research collaboration with Biogen. L.F. is supported by grants from the Dutch Research Council (ZonMW-VIDI 917.14.374 to L.F.), by an ERC Starting Grant, grant agreement 637640 (ImmRisk), by an Oncode Senior Investigator grant and a sponsored research collaboration with Biogen. This project has received funding from the European Research Council (ERC) under the European Union's Horizon 2020 research and innovation programme (grant agreement $\mathrm{n}^{\circ} 772376$ EScORIAL. The authors thank the Biogen CellMap team (Z. Ouyang, N. Bourgeois, E. Lyashenko, P. Cundiff, K. Li, X. Zhang, F. Casey, S. Engle, R. Kleiman, B. Zhang and M. Zavodszky) for the expertise and advice provided toward deriving the cell-type specific expression profiles.

\section{ROSMAP}

The results published here are in whole or in part based on data obtained from the AMP-AD Knowledge Portal (doi:10.7303/syn2580853) Study data were provided by the Rush Alzheimer's Disease Center, Rush University Medical Center, Chicago. Data collection was supported through funding by NIA grants P30AG10161, R01AG15819, R01AG17917, R01AG30146, R01AG36836, U01AG32984, U01AG46152, the Illinois Department of Public Health, and the Translational Genomics Research Institute.

Genotype data: doi:10.1038/mp.2017.20. RNAseq: doi:10.1038/s41593-018-0154-9. snRNAseq: doi:10.7303/syn 18485175

\section{Mayo}

The results published here are in whole or in part based on data obtained from the AMP-AD Knowledge Portal (doi:10.7303/syn2580853). Study data were provided by the following sources: The Mayo Clinic Alzheimer's Disease Genetic Studies, led by Dr. Nilufer Taner and Dr. Steven G. Younkin, Mayo Clinic, Jacksonville, FL using samples from the Mayo Clinic Study of Aging, the Mayo Clinic Alzheimer's Disease Research Center, and the Mayo Clinic Brain Bank. Data collection was supported through funding by NIA grants P50 AG016574, R01 AG032990, U01 AG046139, R01 AG018023, U01 AG006576, U01 AG006786, R01 AG025711, R01 AG017216, R01 AG003949, NINDS grant R01 NS080820, CurePSP Foundation, and support from Mayo Foundation. Study data includes samples collected through the Sun Health Research Institute Brain and Body Donation Program of Sun City, Arizona. The Brain and Body Donation Program is supported by the National Institute of Neurological Disorders and Stroke (U24 NS072026 National Brain and Tissue Resource for Parkinsons Disease and Related Disorders), the National Institute on Aging (P30 AG19610 Arizona Alzheimer's Disease Core Center), the Arizona Department of Health Services (contract 211002, Arizona Alzheimer's Research 
932 Center), the Arizona Biomedical Research Commission (contracts 4001, 0011, 05-901 and 1001

933 to the Arizona Parkinson's Disease Consortium) and the Michael J. Fox Foundation for

934 Parkinson's Research. doi:10.1038/sdata.2016.89

\section{MSBB}

The results published here are in whole or in part based on data obtained from the AMP-AD Knowledge Portal (doi:10.7303/syn2580853). These data were generated from postmortem brain tissue collected through the Mount Sinai VA Medical Center Brain Bank and were provided by Dr. Eric Schadt from Mount Sinai School of Medicine.

\section{CMC}

Data were generated as part of the CommonMind Consortium supported by funding from Takeda Pharmaceuticals Company Limited, F. Hoffman-La Roche Ltd and NIH grants R01MH085542, R01MH093725, P50MH066392, P50MH080405, R01MH097276, RO1-MH-075916, P50M096891, P50MH084053S1, R37MH057881, AG02219, AG05138, MH06692, R01MH1 10921, R01MH109677, R01MH109897, U01MH103392, and contract HHSN271201300031C through IRP NIMH. Brain tissue for the study was obtained from the following brain bank collections: The Mount Sinai NIH Brain and Tissue Repository, the University of Pennsylvania Alzheimer's Disease Core Center, the University of Pittsburgh NeuroBioBank and Brain and Tissue Repositories, and the NIMH Human Brain Collection Core. CMC Leadership: Panos Roussos, Joseph Buxbaum, Andrew Chess, Schahram Akbarian, Vahram Haroutunian (Icahn School of Medicine at Mount Sinai), Bernie Devlin, David Lewis (University of Pittsburgh), Raquel Gur, Chang-Gyu Hahn (University of Pennsylvania), Enrico Domenici (University of Trento), Mette A. Peters, Solveig Sieberts (Sage Bionetworks), Thomas Lehner, Stefano Marenco, Barbara K. Lipska (NIMH).

\section{GTEx}

The Genotype-Tissue Expression (GTEx) Project was supported by the Common Fund of the Office of the Director of the National Institutes of Health (commonfund.nih.gov/GTEx). Additional funds were provided by the NCI, NHGRI, NHLBI, NIDA, NIMH, and NINDS. Donors were enrolled at Biospecimen Source Sites funded by NCILeidos Biomedical Research, Inc. subcontracts to the National Disease Research Interchange (10XS170), Roswell Park Cancer Institute (10XS171), and Science Care, Inc. (X10S172). The Laboratory, Data Analysis, and Coordinating Center (LDACC) was funded through a contract (HHSN268201000029C) to the The Broad Institute, Inc. Biorepository operations were funded through a Leidos Biomedical Research, Inc. subcontract to Van Andel Research Institute (10ST1035). Additional data repository and project management were provided by Leidos Biomedical Research, Inc.(HHSN261200800001E). The Brain Bank was supported supplements to University of Miami grant DA006227. Statistical Methods development grants were made to the University of Geneva (MH090941 \& MH101814), the University of Chicago (MH090951, MH090937, MH101825, \& MH101820), the University of North Carolina - Chapel Hill (MH090936), North Carolina State University (MH101819), Harvard University (MH090948), Stanford University (MH101782), Washington University (MH101810), and to the University of Pennsylvania (MH101822). The datasets used for the analyses described in this manuscript were obtained from dbGaP at http://www.ncbi.nlm.nih.gov/gap through dbGaP accession number phs000424.v7.p2 on $02 / 27 / 2020$. 


\section{NABEC}

980 Data was collected from dbGAP accession phs001301.v1.p1, which was generated by J. R. Gibbs, M. van der Brug, D. Hernandez, B. Traynor, M. Nalls, S-L. Lai, S. Arepalli, A. Dillman, I. Rafferty, J. Troncoso, R. Johnson, H. R. Zielke, L. Ferrucci, D. Longo, M.R. Cookson, and A.B. Singleton. The NABEC dataset was generated at National Institute on Aging, Bethesda, MD, USA, Institute of Neurology, University College London, London, UK, The Scripps Research Institute, Jupiter, FL, USA, Johns Hopkins University, Baltimore, MD, USA, and the University of Maryland Medical School, Baltimore, MD, USA. NABEC was funded by Z01 AG000949-02. National Institutes of Health, Bethesda, MD, USA and Z01 AG000015-49. National Institutes of Health, Bethesda, MD, USA.

\section{TargetALS}

This data set was generated and supported by the following: Target ALS Human Postmortem Tissue Core, New York Genome Center for Genomics of Neurodegenerative Disease, Amyotrophic Lateral Sclerosis Association and TOW Foundation.

\section{Braineac}

Data was collected from doi.org/10.1038/s41467-020-14483-x, which was generated by Mina Ryten, David Zhang, and Karishma D'Sa, Sebastian Guelfi and Regina Reynolds. Mina Ryten, David Zhang, and Karishma D'Sa were supported by the UK Medical Research Council (MRC) through the award of Tenure-track Clinician Scientist Fellowship to Mina Ryten

1000

1001

1002

1003

1004

1005

1006

1007 (MR/N008324/1). Sebastian Guelfi was supported by Alzheimer's Research UK through the award of a PhD Fellowship (ARUK-PhD2014-16). Regina Reynolds was supported through the award of a Leonard Wolfson Doctoral Training Fellowship in Neurodegeneration. All RNA sequencing data performed as part of this study were generated by the commercial company AROS Applied Biotechnology A/S (Denmark).

We also would like to thank Guelfi et al. ${ }^{119}$ for the use of their data.

1010

1011

\section{European Nucleotide Archive}

We would like to thank all donors and their families, principal investigators and their funding bodies for each of the projects included from the European Nucleotide Archive.

\section{UCLA ASD, Bipseq, BrainGVEx and LIBD}

Data were generated as part of the PsychENCODE Consortium supported by: U01MH103339, U01MH103365, U01MH103392, U01MH103340, U01MH103346, R01MH105472, R01MH094714, R01MH105898, R21MH102791, R21MH105881, R21MH103877, and P50MH106934 awarded to: Schahram Akbarian (Icahn School of Medicine at Mount Sinai), Gregory Crawford (Duke), Stella Dracheva (Icahn School of Medicine at Mount Sinai), Peggy Farnham (USC), Mark Gerstein (Yale), Daniel Geschwind (UCLA), Thomas M. Hyde (LIBD), Andrew Jaffe (LIBD), James A. Knowles (USC), Chunyu Liu (UIC), Dalila Pinto (Icahn School of Medicine at Mount Sinai), Nenad Sestan (Yale), Pamela Sklar (Icahn School of Medicine at Mount Sinai), Matthew State (UCSF), Patrick Sullivan (UNC), Flora Vaccarino (Yale), Sherman Weissman (Yale), Kevin White (UChicago) and Peter Zandi (JHU). 


\section{Author contributions}

1027 N.K., O.E.G, and H.W. processed the RNA-seq and genotype data. N.K. and H.W. were

1028 responsible for data management. N.K. and H.W. were responsible for the cis-eQTL analysis.

1029 H.W. was responsible for the trans-eQTL analysis. M.V., Z.O. and M.I.Z. were responsible for

1030 the cell type proportion prediction. N.K. and M.V. were responsible for the cell type interaction

1031 analysis. S.D. was responsible for the selection of brain samples from ENA. D.B., Y.H., C.-Y.C.,

1032 E.E.M, T.R.G. and E.A.T. were responsible for MR and colocalization analysis and

1033 interpretation. P.D., O.B.B. and L.F. were responsible for the Downstreamer analysis. L.F.,

1034 E.A.T. and H.R. acquired funding and supervised the study. N.K., E.A.T., M.V., D.B, Y.H., C.-

1035 Y.C., O.B.B., H.R., L.F. and H.W. drafted the manuscript. All authors have proof-read the 1036 manuscript. 


\section{References}

1. Vos, T. et al. Global burden of 369 diseases and injuries in 204 countries and territories, 1990-2019: a systematic analysis for the Global Burden of Disease Study 2019. The Lancet 396,

1043 1204-1222 (2020).

10442 2. World Alzheimer Report 2018 - The state of the art of dementia research: New frontiers.

1045 NEW Front. 48.

1046 3. Donovan, M. K. R., D’Antonio-Chronowska, A., D’Antonio, M. \& Frazer, K. A. Cellular 1047 deconvolution of GTEx tissues powers discovery of disease and cell-type associated regulatory 1048 variants. Nat. Commun. 11, 955 (2020).

1049 4. Wang, D. et al. Comprehensive functional genomic resource and integrative model for 1050 the human brain. Science 362, (2018).

1051 5. Raj, T. et al. Integrative transcriptome analyses of the aging brain implicate altered 1052 splicing in Alzheimer's disease susceptibility. Nat. Genet. 50, 1584-1592 (2018).

1053 6. Hodes, R. J. \& Buckholtz, N. Accelerating Medicines Partnership: Alzheimer's Disease 1054 (AMP-AD) Knowledge Portal Aids Alzheimer's Drug Discovery through Open Data Sharing. 1055 Expert Opin. Ther. Targets 20, 389-391 (2016).

1056 7. Ramasamy, A. et al. Genetic variability in the regulation of gene expression in ten 1057 regions of the human brain. Nat. Neurosci. 17, 1418-1428 (2014).

1058 8. Consortium*, T. P. Revealing the brain's molecular architecture. Science 362, 1262-1263 1059 (2018).

10609 9. Fromer, M. et al. Gene expression elucidates functional impact of polygenic risk for 1061 schizophrenia. Nat. Neurosci. 19, 1442-1453 (2016).

1062 10. BrainSeq: A Human Brain Genomics Consortium. BrainSeq: Neurogenomics to Drive 1063 Novel Target Discovery for Neuropsychiatric Disorders. Neuron 88, 1078-1083 (2015).

1064 11. Gibbs, J. R. et al. Abundant Quantitative Trait Loci Exist for DNA Methylation and Gene 1065 Expression in Human Brain. PLOS Genet. 6, e1000952 (2010).

1066 12. Prudencio, M. et al. Distinct brain transcriptome profiles in C9orf72-associated and 1067 sporadic ALS. Nat. Neurosci. 18, 1175-1182 (2015).

1068 13. Leinonen, R. et al. The European Nucleotide Archive. Nucleic Acids Res. 39, D28-D31 1069 (2011).

1070

1071 14. Võsa, U. et al. Unraveling the polygenic architecture of complex traits using blood eQTL metaanalysis. bioRxiv 447367 (2018) doi:10.1101/447367.

1072 15. Dobbyn, A. et al. Landscape of Conditional eQTL in Dorsolateral Prefrontal Cortex and 1073 Co-localization with Schizophrenia GWAS. Am. J. Hum. Genet. 102, 1169-1184 (2018).

1074

1075

1076

1077

1078

1079 16. Wingender, E., Dietze, P., Karas, H. \& Knüppel, R. TRANSFAC: a database on transcription factors and their DNA binding sites. Nucleic Acids Res. 24, 238-241 (1996).

17. Võsa, U. et al. Unraveling the polygenic architecture of complex traits using blood eQTL metaanalysis. bioRxiv 447367 (2018) doi:10.1101/447367.

18. Foley, C. N. et al. A fast and efficient colocalization algorithm for identifying shared genetic risk factors across multiple traits. Nat. Commun. 12, 764 (2021).

1080 19. Fu, J. et al. Unraveling the Regulatory Mechanisms Underlying Tissue-Dependent 1081 Genetic Variation of Gene Expression. PLOS Genet. 8, e1002431 (2012).

1082 20. Fu, J. et al. Unraveling the Regulatory Mechanisms Underlying Tissue-Dependent 1083 Genetic Variation of Gene Expression. PLOS Genet. 8, e1002431 (2012). 
1084

21. Glastonbury, C. A., Couto Alves, A., El-Sayed Moustafa, J. S. \& Small, K. S. Cell-Type Heterogeneity in Adipose Tissue Is Associated with Complex Traits and Reveals DiseaseRelevant Cell-Specific eQTLs. Am. J. Hum. Genet. 104, 1013-1024 (2019).

22. Raúl Aguirre-Gamboa et al. Deconvolution of bulk blood eQTL effects into immune cell subpopulations. BMC Bioinformatics 21, 243 (2020).

23. Zhengyu, O. et al. CellMap: Characterizing the type and composition of iPSC-derived cell lines from bulk RNA-seq data. Manuscr. Prep.

24. BAHNEY, J. \& VON BARTHELD, C. S. The Cellular Composition and Glia-Neuron Ratio in the Spinal Cord of a Human and a Non-Human Primate: Comparison with other Species and Brain Regions. Anat. Rec. Hoboken NJ 2007 301, 697-710 (2018).

25. Patrick, E. et al. Deconvolving the contributions of cell-type heterogeneity on cortical gene expression. PLOS Comput. Biol. 16, e1008120 (2020).

26. Herculano-Houzel, S. The human brain in numbers: a linearly scaled-up primate brain. Front. Hum. Neurosci. 3, (2009).

27. von Bartheld, C. S., Bahney, J. \& Herculano-Houzel, S. The Search for True Numbers of Neurons and Glial Cells in the Human Brain: A Review of 150 Years of Cell Counting. J. Comp. Neurol. 524, 3865-3895 (2016).

28. Mathys, H. et al. Single-cell transcriptomic analysis of Alzheimer's disease. Nature 570, 332-337 (2019).

29. Ng, B. et al. Using Transcriptomic Hidden Variables to Infer Context-Specific Genotype Effects in the Brain. Am. J. Hum. Genet. 105, 562-572 (2019).

30. Zhao, B. et al. Large-scale GWAS reveals genetic architecture of brain white matter microstructure and genetic overlap with cognitive and mental health traits $(\mathrm{n}=17,706)$. Mol. Psychiatry 1-13 (2019) doi:10.1038/s41380-019-0569-z.

31. Smorodchenko, A. et al. Comparative analysis of uncoupling protein 4 distribution in various tissues under physiological conditions and during development. Biochim. Biophys. Acta BBA - Biomembr. 1788, 2309-2319 (2009).

32. Liu, D. et al. Mitochondrial UCP4 mediates an adaptive shift in energy metabolism and increases the resistance of neurons to metabolic and oxidative stress. Neuromolecular Med. $\mathbf{8}$, 389-414 (2006).

33. Yasuno, K. et al. Synergistic association of mitochondrial uncoupling protein (UCP) genes with schizophrenia. Am. J. Med. Genet. Part B Neuropsychiatr. Genet. Off. Publ. Int. Soc. Psychiatr. Genet. 144B, 250-253 (2007).

34. Ho, P. W. et al. Mitochondrial neuronal uncoupling proteins: a target for potential disease-modification in Parkinson's disease. Transl. Neurodegener. 1, 3 (2012).

35. Ramsden, D. B. et al. Human neuronal uncoupling proteins 4 and 5 (UCP4 and UCP5): structural properties, regulation, and physiological role in protection against oxidative stress and mitochondrial dysfunction. Brain Behav. 2, 468-478 (2012).

36. Giambartolomei, C. et al. Bayesian test for colocalisation between pairs of genetic association studies using summary statistics. PLoS Genet. 10, e1004383 (2014).

37. Consortium*†, I. M. S. G. Multiple sclerosis genomic map implicates peripheral immune cells and microglia in susceptibility. Science 365, (2019).

38. International Multiple Sclerosis Genetics, C. et al. Genetic risk and a primary role for cell-mediated immune mechanisms in multiple sclerosis. Nature 476, 214-9 (2011).

39. Consortium*†, I. M. S. G. Multiple sclerosis genomic map implicates peripheral immune cells and microglia in susceptibility. Science 365, (2019). 
40. Jones, G., Prosser, D. E. \& Kaufmann, M. 25-Hydroxyvitamin D-24-hydroxylase (CYP24A1): its important role in the degradation of vitamin D. Arch Biochem Biophys 523, 9-18

1132 (2012).

41. Schlingmann, K. P. et al. Mutations in CYP24A1 and idiopathic infantile hypercalcemia. N Engl J Med 365, 410-21 (2011). 42. Cappellani, D. et al. Hereditary Hypercalcemia Caused by a Homozygous Pathogenic Variant in the CYP24A1 Gene: A Case Report and Review of the Literature. Case Rep Endocrinol 2019, 4982621 (2019). D deficiency and its role in neurological conditions: A review. Rev. Neurol. (Paris) 172, 109122 (2016).

44. Agnello, L. et al. CYP27A1, CYP24A1, and RXR-alpha Polymorphisms, Vitamin D, and Multiple Sclerosis: a Pilot Study. J Mol Neurosci 66, 77-84 (2018). 45. Pierrot-Deseilligny, C. \& Souberbielle, J. C. Is hypovitaminosis D one of the environmental risk factors for multiple sclerosis? Brain 133, 1869-88 (2010). 46. Rhead, B. et al. Mendelian randomization shows a causal effect of low vitamin D on multiple sclerosis risk. Neurol. Genet. 2, e97 (2016).

47. Jacobs, B. M., Noyce, A. J., Giovannoni, G. \& Dobson, R. BMI and low vitamin D are causal factors for multiple sclerosis: A Mendelian Randomization study. Neurol. Neuroimmunol. Neuroinflammation 7, (2020). 48. Jiang, X., Ge, T. \& Chen, C.-Y. The causal role of circulating vitamin D concentrations in human complex traits and diseases: a large-scale Mendelian randomization study. Sci. Rep. 11, 184 (2021).

49. Ramasamy, A. et al. Genetic evidence for a pathogenic role for the vitamin D3 metabolizing enzyme CYP24A1 in multiple sclerosis. Mult. Scler. Relat. Disord. 3, 211-219 (2014). 50. van Luijn, M. M. et al. Multiple sclerosis-associated CLEC16A controls HLA class II expression via late endosome biogenesis. Brain J. Neurol. 138, 1531-1547 (2015). 51. Ferrari, R. et al. Frontotemporal dementia and its subtypes: a genome-wide association study. Lancet Neurol 13, 686-99 (2014). 52. Wray, N. R. et al. Genome-wide association analyses identify 44 risk variants and refine the genetic architecture of major depression. Nat. Genet. 50, 668-681 (2018).

53. Li, Z. et al. Genetic variants associated with Alzheimer's disease confer different cerebral cortex cell-type population structure. Genome Med. 10, 43 (2018).

54. Park, Y. et al. Single-cell deconvolution of 3,000 post-mortem brain samples for eQTL and GWAS dissection in mental disorders. bioRxiv 2021.01.21.426000 (2021) doi:10.1101/2021.01.21.426000.

55. Li, Z. et al. The TMEM106B FTLD-protective variant, rs1990621, is also associated with increased neuronal proportion. Acta Neuropathol. (Berl.) 139, 45-61 (2020). 56. Raudvere, U. et al. g:Profiler: a web server for functional enrichment analysis and conversions of gene lists (2019 update). Nucleic Acids Res. 47, W191-W198 (2019). 57. Sankaran, V. G. et al. Human Fetal Hemoglobin Expression Is Regulated by the Developmental Stage-Specific Repressor BCL11A. Science 322, 1839-1842 (2008). 58. Jiang, J. et al. cMYB is involved in the regulation of fetal hemoglobin production in adults. Blood 108, 1077-1083 (2006). 
1175 59. Métais, J.-Y. et al. Genome editing of HBG1 and HBG2 to induce fetal hemoglobin. 1176 Blood Adv. 3, 3379-3392 (2019).

1177 60. J, D. \& A, B. Dalfampridine: a brief review of its mechanism of action and efficacy as a 1178 treatment to improve walking in patients with multiple sclerosis. Curr. Med. Res. Opin. 27, 1179 1415-1423 (2011).

1180 61. Al-Owais, M. M. et al. Multiple mechanisms mediating carbon monoxide inhibition of 1181 the voltage-gated K + channel Kv1.5. Cell Death Dis. 8, e3163-e3163 (2017).

1182 62. Rus, H. et al. The voltage-gated potassium channel Kv1.3 is highly expressed on 1183 inflammatory infiltrates in multiple sclerosis brain. Proc. Natl. Acad. Sci. 102, 11094-11099 1184 (2005).

1185 63. Noordam, R. et al. Multi-ancestry sleep-by-SNP interaction analysis in 126,926 1186 individuals reveals lipid loci stratified by sleep duration. Nat. Commun. 10, 5121 (2019).

1187 64. Gallois, A. et al. A comprehensive study of metabolite genetics reveals strong pleiotropy 1188 and heterogeneity across time and context. Nat. Commun. 10, 4788 (2019).

1189 65. Sun, B. B. et al. Genomic atlas of the human plasma proteome. Nature 558, 73-79 1190 (2018).

1191 66. Suhre, K. et al. Connecting genetic risk to disease end points through the human blood 1192 plasma proteome. Nat. Commun. 8, 14357 (2017).

1193 67. Tin, A. et al. Target genes, variants, tissues and transcriptional pathways influencing 1194 human serum urate levels. Nat. Genet. 51, 1459-1474 (2019).

1195 68. Kichaev, G. et al. Leveraging Polygenic Functional Enrichment to Improve GWAS 1196 Power. Am. J. Hum. Genet. 104, 65-75 (2019). 69. Pers, T. H. et al. Biological interpretation of genome-wide association studies using predicted gene functions. Nat. Commun. 6, 5890 (2015). 70. Deelen, P. et al. Improving the diagnostic yield of exome- sequencing by predicting gene-phenotype associations using large-scale gene expression analysis. Nat. Commun. 10, 1-13 (2019).

71. Ripke, S. et al. Biological insights from 108 schizophrenia-associated genetic loci. Nature 511, 421-427 (2014).

72. Nalls, M. A. et al. Identification of novel risk loci, causal insights, and heritable risk for Parkinson's disease: a meta-analysis of genome-wide association studies. Lancet Neurol 18, 1091-1102 (2019).

73. Kunkle, B. W. et al. Genetic meta-analysis of diagnosed Alzheimer's disease identifies new risk loci and implicates $\mathrm{A} \beta$, tau, immunity and lipid processing. Nat. Genet. 51, 414-430 (2019).

1210 74. Conroy, J. et al. A novel locus for episodic ataxia:UBR4 the likely candidate. Eur. J. Hum. Genet. EJHG 22, 505-510 (2014).

$121476 . \quad$ Kalinowska-Lyszczarz, A. \& Losy, J. The Role of Neurotrophins in Multiple Sclerosis1215 Pathological and Clinical Implications. Int. J. Mol. Sci. 13, 13713-13725 (2012).

1216 77. Kerschensteiner, M. et al. Activated human T cells, B cells, and monocytes produce brain-derived neurotrophic factor in vitro and in inflammatory brain lesions: a neuroprotective role of inflammation? J. Exp. Med. 189, 865-870 (1999). 
78. De Santi, L. et al. Neuroinflammation and neuroprotection: an update on (future) neurotrophin-related strategies in multiple sclerosis treatment. Curr. Med. Chem. 18, 1775-1784 (2011). Immunotherapy. Neuron 97, 742-768 (2018). 80. Redondo, J. et al. Purkinje Cell Pathology and Loss in Multiple Sclerosis Cerebellum. Brain Pathol. Zurich Switz. 25, 692-700 (2015). 81. Wang, X. \& Goldstein, D. B. Enhancer Domains Predict Gene Pathogenicity and Inform Gene Discovery in Complex Disease. Am. J. Hum. Genet. 106, 215-233 (2020). 82. Wijst, M. G. P. van der et al. Single-cell RNA sequencing identifies celltype-specific ciseQTLs and co-expression QTLs. Nat. Genet. 50, 493-497 (2018). differentiation | bioRxiv. https://www.biorxiv.org/content/10.1101/2020.05.21.103820v1. 84. Consortium, S. W. G. of the P. G., Ripke, S., Walters, J. T. \& O'Donovan, M. C. Mapping genomic loci prioritises genes and implicates synaptic biology in schizophrenia. medRxiv 2020.09.12.20192922 (2020) doi:10.1101/2020.09.12.20192922.

85. Davenport, E. E. et al. Discovering in vivo cytokine-eQTL interactions from a lupus clinical trial. Genome Biol. 19, 168 (2018).

86. Genetic effects on gene expression across human tissues. Nature 550, 204-213 (2017).

87. Hemani, G. et al. The MR-Base platform supports systematic causal inference across the human phenome. eLife 7, (2018). 88. Hemani, G., Bowden, J. \& Davey Smith, G. Evaluating the potential role of pleiotropy in Mendelian randomization studies. Hum. Mol. Genet. 27, R195-R208 (2018).

89. Eliciting priors and relaxing the single causal variant assumption in colocalisation analyses. https://journals.plos.org/plosgenetics/article?id=10.1371/journal.pgen.1008720. 90. Team, T. S. E. synapseclient: A client for Synapse, a collaborative compute space that allows scientists to share and analyze data together.

91. Tsui, B., Dow, M., Skola, D. \& Carter, H. Extracting allelic read counts from 250,000 human sequencing runs in Sequence Read Archive. bioRxiv 386441 (2018) doi:10.1101/386441. 92. Swertz, M. A. et al. The MOLGENIS toolkit: rapid prototyping of biosoftware at the push of a button. BMC Bioinformatics 11, S12 (2010).

1254 95. Anders, S., Pyl, P. T. \& Huber, W. HTSeq-a Python framework to work with high93. Frankish, A. et al. GENCODE reference annotation for the human and mouse genomes. Nucleic Acids Res. 47, D766-D773 (2019).

94. Dobin, A. et al. STAR: ultrafast universal RNA-seq aligner. Bioinformatics 29, 15-21 (2013). throughput sequencing data. Bioinformatics 31, 166-169 (2015).

96. Robinson, M. D. \& Oshlack, A. A scaling normalization method for differential expression analysis of RNA-seq data. Genome Biol. 11, R25 (2010).

97. Robinson, M. D., McCarthy, D. J. \& Smyth, G. K. edgeR: a Bioconductor package for differential expression analysis of digital gene expression data. Bioinformatics 26, 139-140 (2010).

98. R Core Team. R: A language and environment for statistical computing. (R Foundation for Statistical Computing, 2017).

1264 Sequence Data. https://www.bioinformatics.babraham.ac.uk/projects/fastqc/. 
100. Broad Institute. Picard Tools. (2019).

1266 101. McCarthy, S. et al. A reference panel of 64,976 haplotypes for genotype imputation. Nat.

1267 Genet. 48, 1279-1283 (2016).

1268 102. Deelen, P. et al. Genotype harmonizer: automatic strand alignment and format conversion for genotype data integration. BMC Res. Notes 7, 901 (2014). 103. Das, S. et al. Next-generation genotype imputation service and methods. Nat. Genet. $\mathbf{4 8 ,}$ 1284-1287 (2016).

1272 104. Chang, C. C. et al. Second-generation PLINK: rising to the challenge of larger and richer datasets. GigaScience 4, (2015).

105. Roberts, T. C., Morris, K. V. \& Wood, M. J. A. The role of long non-coding RNAs in neurodevelopment, brain function and neurological disease. Philos. Trans. R. Soc. B Biol. Sci. 369, (2014).

106. Westra, H.-J. et al. Systematic identification of trans-eQTLs as putative drivers of known disease associations. Nat. Genet. 45, 1238-1243 (2013).

107. Lyon, M. et al. The variant call format provides efficient and robust storage of GWAS summary statistics. bioRxiv 2020.05.29.115824 (2020) doi:10.1101/2020.05.29.115824.

108. Buniello, A. et al. The NHGRI-EBI GWAS Catalog of published genome-wide association studies, targeted arrays and summary statistics 2019. Nucleic Acids Res. 47, D1005D1012 (2019).

109. Lawson, C. L. \& Hanson, R. J. Solving Least Squares Problems. (Society for Industrial and Applied Mathematics, 1995). doi:10.1137/1.9781611971217.

110. Virtanen, P. et al. SciPy 1.0: fundamental algorithms for scientific computing in Python. Nat. Methods 17, 261-272 (2020).

111. Mathys, H. et al. Single-cell transcriptomic analysis of Alzheimer's disease. Nature 570, 332-337 (2019).

112. Stuart, T. et al. Comprehensive Integration of Single-Cell Data. Cell 177, 1888-1902.e21 (2019).

113. Hafemeister, C. \& Satija, R. Normalization and variance stabilization of single-cell RNAseq data using regularized negative binomial regression. Genome Biol. 20, 296 (2019).

114. McInnes, L., Healy, J. \& Melville, J. UMAP: Uniform Manifold Approximation and Projection for Dimension Reduction. ArXiv180203426 Cs Stat (2020).

115. Mathys, H. et al. Single-cell transcriptomic analysis of Alzheimer's disease. Nature 570, 332-337 (2019).

116. Bonett, D. G. \& Wright, T. A. Sample size requirements for estimating pearson, kendall and spearman correlations. Psychometrika 65, 23-28 (2000).

1300 117. Elsworth, B. et al. The MRC IEU OpenGWAS data infrastructure. bioRxiv

1301

1302 118. $\mathrm{Ng}$, B. et al. An xQTL map integrates the genetic architecture of the human brain's

1303 transcriptome and epigenome. Nat. Neurosci. 20, 1418-1426 (2017).

1304 119. Guelfi, S. et al. Regulatory sites for splicing in human basal ganglia are enriched for 1305 disease-relevant information. Nat. Commun. 11, 1041 (2020). 


\section{Figure Legends}

1308 Figure 1. Overview of the study. We downloaded publicly available RNA-seq and genotype data from 15 different datasets consisting 8,727 RNA-seq measurements from 7 main brain regions in 6,518 individuals. We performed cis-, trans- and interaction-eQTL analysis, built a brain-specific gene coregulation network and prioritized genes using Mendelian randomization, colocalization and the co-regulation network.

Figure 2. Overview of the datasets. (A) The number of samples per included cohort, with each color representing one of the 7 major brain regions. (B) The number of genotypes per cohort, with each color representing a population. (C) The number of individuals per cohort, with each color representing an eQTL dataset. The number of individuals is different from the intersection between the number of RNA-seq samples and number of genotypes, because not all samples with genotypes have RNA-seq samples and vice-versa, and some individuals with genotypes have multiple RNA-seq measurements. (D) PCA dimensionality reduction plot of the normalized expression data after covariate correction. Each dot represents an RNA-seq sample and is colored by brain region. The figure shows that the samples cluster mainly on brain region.

Figure 3. Conditional cis-eQTLs. (A) The number of conditional cis-eQTLs per eQTL dataset. (B) Comparison of characteristics between primary and non-primary eQTLs, where each row compares the eQTL genes for that rank with eQTL genes from the previous rank. P-values are calculated using a Wilcoxon test between significant and non-significant genes. (left) The difference in mean gene expression levels; (middle) the difference in distance between the most significant SNP-gene combination and the transcription start site (TSS); (right) the difference in probability for loss of function intolerance (pLI) score. For primary, secondary and quaternary eQTLs, non-significant eQTLs have higher pLI scores. (C) Replication of primary cis-eQTLs between the cortex eQTLs of different ethnicities and (D) the different brain regions for the European datasets. $\mathrm{n}$ indicates sample size of each dataset. Numbers in boxes indicate the number of eQTLs that are significant in both the discovery and the replication dataset, and the percentage of those that shows the same direction of effect. (E) Replication of primary ciseQTLs of Cortex-EUR (discovery) in all the GTEx tissues (replication). Each dot is a different GTEx tissue, the $\mathrm{x}$-axis is the number of eQTLs that is significant in both discovery and

Figure 4. Cell type interacting eQTLs. (A) Spearman correlations between the 5 predicted cell count proportions. Lower triangle is within cortex samples, upper triangle is within cerebellum samples. (B) Predicted cell type proportions (x-axis) compared to cell type proportions measured using immunohistochemistry (IHC; y-axis) for 42 ROSMAP samples. Values in the plot are Pearson correlation coefficients. Cell count predictions for most cell types closely approximates actual IHC cell counts, although neurons are underestimated. (C) Number of cell type interacting eQTLs for Cortex-EUR deconvoluted cell types. The majority of interactions are with neurons 
1347 and oligodendrocytes. Notably, most interactions are unique for one cell type in $90 \%$ of the 1348 cases. (D, E, F) Replication of cell type interacting eQTLs for STMN4 (D), FAM221A (E) and SLC25A27 (F), consisting of the scatterplot of the interaction eQTL in MetaBrain Cortex-EUR bulk RNA-seq (left) and a forest plot for the eQTL effect in the ROSMAP snRNA-seq data (right). Scatterplot: the $\mathrm{x}$-axis shows the estimated cell type proportion, the y-axis shows the gene expression, each dot represents a sample. Colors indicate SNP genotype, with yellow being the minor allele. Values under the alleles are Spearman correlation coefficients. Forest plot: Spearman coefficients with effect direction relative to the minor allele when replicating the eQTL effect in ROSMAP single nucleus data $(n=38)$. Error bars indicate $95 \%$ confidence interval. Each row denotes a cell type specific dataset: excitatory neurons (EX), oligodendrocytes (OLI), inhibitory neurons (IN), astrocytes (AST), oligodendrocyte precursor cells (OPC), microglia (MIC), pericytes (PER) and endothelial cells (END). Cell types highlighted in bold reflect the equivalent to the cell type used in the interaction eQTL.

Figure 5. Mendelian randomization and colocalization of brain-related traits. (A) Number of significant Mendelian randomization (MR) signals (blue) and those with both MR and Coloc significant signals for 15 brain-related traits. (B) SNP and effect allele (EA), eQTL beta and GWAS odds ratio for 20 multiple sclerosis (MS) genes that are both MR and Coloc significant, and their Wald ratio p-value. Cell type interaction eQTL for CYP24A1 (D) and CLECL1 (E), showing interactions with predicted neuron, and macrophage proportions respectively. The $\mathrm{x}-$ axis shows the estimated cell type proportion, the y-axis shows the gene expression, each dot represents a sample. Colors indicate SNP genotype, with yellow being the MS risk allele. Values under the alleles are Spearman correlation coefficients.

Figure 6. Trans-eQTLs in brain. (A) Location of identified trans-eQTLs, with the SNP position (x-axis) and gene position (y-axis) in the genome. Size of the dots indicate the p-value of the trans-eQTL (larger is more significant). 7p21.3, the locus with most (83\%) of the transeQTLs, is highlighted. (B) Three SNPs in the 7p21.3 locus and the number of datasets and number of up- and down-regulated trans-eQTL genes each SNP has. For rs1990622, a SNP associated with frontotemporal lobar degeneration, the 35 genes it affects in trans and the 1 gene it affects in cis are shown. (C) Two examples of convergent effects, where multiple independent SNPs affect the same genes in trans. Left: trans-eQTLs of rs1427407 and rs4895441 on HBG2

1381 Figure 7. Gene co-regulation (A) Genes that are co-regulated with genes that are within amyotrophic lateral sclerosis (ALS) loci. Co-regulation scores between genes are calculated using all MetaBrain samples, MetaBrain cerebellum samples, or MetaBrain cortex samples. Except for URB4, cortex and cerebellum networks find different co-regulated genes for ALS. (B) Co-regulation network using all MetaBrain samples for all genes prioritized for ALS by Downstreamer. (C) Top 5 Human Phenotype Ontology (HPO) enrichments for the 
1387 Downstreamer prioritized ALS genes. (D) Genes that are co-regulated with genes that are within 1388 multiple sclerosis loci. Co-regulation scores between genes are calculated using a heterogeneous 1389 multi-tissue network, MetaBrain cerebellum samples, or MetaBrain cortex samples. Most genes 1390 are found using a large heterogenous co-regulation network. (E) Co-regulation network of all 1391 MetaBrain samples for 33 genes prioritized by Downstreamer in cortex. Colors indicate the 1392 neutrophin signaling pathway enrichment Z-scores. (F) Top 5 KEGG enrichments for the 1393 Downstreamer prioritized multiple sclerosis genes in cortex. 


\section{Supplementary Figure Legends}

Supplementary Figure 1. European Nucleotide Archive brain sample selection. (A) Principal component (PC) analysis on the expression data of 74,052 samples included in the SkyMap database shows clustering on tissue type but also many outliers with high PC1 scores. (B) Coloring on single and paired-end sequencing shows no clear clustering. (C) Coloring single cell identifies the samples with high PC1 scores as single-cell samples. (D) Mean \% reads mapped, number of reads, and max reads per bin of PC1. (E) Re-calculation of PCs on all samples with PC score $<0$ in panel A-D, after covariate correction. (F) Brain and Tissue score calculated by correlating expression of known tissue and brain samples to each of the PCs. (G) As panel F, cancer score was calculated by correlating expression of known cancer genes to all PCs.

Supplementary Figure 2. RNA-seq alignment QC. The two main RNA-seq QC metrics used for filtering samples. (A) Percentage coding bases colored by dataset and (B) percentage of reads aligned colored per dataset. Red dotted line is the threshold for filtering ( $10 \%$ for coding bases and $60 \%$ for percentage reads aligned respectively). Triangles are samples filtered out by any of the RNA-seq QC metrics.

Supplementary Figure 3. Sample filtering by PCA. Principal component analysis (PCA) plot before normalization and covariate removal. For all plots the red line indicates 4 standard deviations from the mean and red dots are samples to be filtered out. (A) PCA on all samples after removing alignment QC outliers. (B) PCA on samples after removal of outlier samples from A. (C) PCA on samples after removal of outlier samples of A and B.

Supplementary Figure 4. PCA before and after covariate correction. (A) PC1 and PC2 on normalized expression data before covariate correction, colored on dataset. (B) PC1 and PC2 on normalized expression data after covariate correction.

Supplementary Figure 5. Assigning ethnicity through principal component analysis. For each of the included datasets principal component (PC) scores are calculated on their genotypes. Samples are clustered with the 1000 genome samples (left). Right panels show dataset genotype samples without $1000 \mathrm{~g}$ samples on the right projected on the same PCs. Using k-nearest neighbors clustering, samples are assigned an ethnicity based on their closeness to the $1000 \mathrm{~g}$ samples of a population. 
Supplementary Figure 6. eQTL Z-score comparison between datasets. The pairwise spearman correlation and concordance of direction of the eQTL Z-scores between all cohorts, and between each cohort and the meta-analysis Z-score. As two examples, (A) shows the Z-score comparison between Cortex-EUR eQTL datasets EUR-LIBD_h650 and EUR-UCLA_ASD, and (B) shows the Z-score comparison between the meta-analysis Z-score and the Cortex-EUR cohort EUR-AMPAD-ROSMAP-V2. (C) shows the correlation for each pairwise combination of cohorts between each other (small dots), and with the meta-analysis Z-scores (large dots). (D) shows the directional concordance for each pairwise combination of cohorts between each other (small dots), and with the meta-analysis Z-scores (large dots). The dots in (C) and (D) that correspond to the (A) and (B) plots are shown by the grey dottes lines. of reads mapped to the MAPT gene located on the primary assembly (ENSG00000186868) and the MAPT genes located on the patch chromosomes (ENSG00000276155 and ENSG00000277956). Each dot is an individual, and the color shows if they are homozygous reference (0/0), heterozygous (0/1), or homozygous alternative (1/1) for a SNP (rs34619181) located in the MAPT gene. Left plot compares counts mapped to ENSG00000186868 (ref) to those mapped to ENSG00000276155 (patch), middle plot compares ENSG00000186868 (ref) and ENSG00000277956 (patch), right plot compares ENSG00000276155 (patch) and ENSG00000277956 (patch). locus (X-axis) for all the datasets used in the Cortex-EUR meta-analysis. Left upper plot shows the meta-analysis Z-score. Blue dots are the SNPs that are in high LD with the top SNP.

Supplementary Figure 9. Colocalization locus plot for MAPT. Y-axis shows the colocalization $\log 10(-p$-value). X-axis shows the position of the SNPs (dots). Color is the LD with rs56240678.

Supplementary Figure 10. (A) Mean of $\log _{2}$ of the expression (x-axis) and standard deviation of the $\log _{2}$ of expression for primary, secondary, tertiary, and quaternary eQTL genes. eQTLs that have only one independent SNP effect have higher mean expression but lower standard deviation than genes with multiple independent effects. (B) g:profiler enrichment for all genes with a single independent eQTL effect. (C) g:profiler enrichment for all genes with multiple independent eQTL effects. 
$\log 2(\mathrm{TMM}+1)$ expression in cortex (x-axis) and cerebellum (y-axis) of the 846 eQTL genes that were only significant in cerebellum. Blue line is the minima of the bimodal distribution and is used as cut-off point in panel $\mathbf{C}(\mathbf{C})$ The expression in cortex (x-axis) and cerebellum (y-axis) of the 846 eQTL genes that were only significant eQTLs in cerebellum. The blue line is the cut-off from panel B. (D) The expression (dots) and standard deviation (lines) of the transcription factors that are enriched for binding to transcription sites around the 662 genes for cortex ( $\mathrm{x}$ axis) and cerebellum (y-axis). The 5 transcription factors that are labelled are lower expressed in cortex and higher expressed in cerebellum.

Supplementary Figure 12. Cortex primary eQTL replication in GTEx. The replication between primary cis-eQTLs of Cortex-EUR (discovery) with all the GTEx tissues (replication). The $\mathrm{x}$-axis is the number of eQTLs that is significant in both discovery and replication, and the $y$-axis is the percentage that shows the same direction of effect.

Supplementary Figure 13. Comparison of meta-analysis Z-scores for eQTLs detected in the different MetaBrain datasets (x-axis), and eQTLgen (y-axis).

Supplementary Figure 14. Distribution of predicted cell proportions. The distribution of the predicted cell proportions (x-axis) for cortex and cerebellum samples (y-axis). exception of the spinal cord. Visualization of the cell type proportions with one row per cell type and colors indicating brain region. (A) Density plot where the $\mathrm{x}$-axis shows the predicted cell type proportion, and the y-axis shows the frequency. (B) Boxplot of the predicted cell type proportion. Boxes represent the $25^{\text {th }}$ and $75^{\text {th }}$ percentiles and internal line represents the median. The whiskers represent 1.5 multiplied by the inter-quartile range. Outliers are shown as individual points. the exception of the spinal cord. Visualization of the cell type proportions with one row per brain region and colors indicating cell types. (A) Density plot where the $\mathrm{x}$-axis shows the predicted cell type proportion, and the y-axis shows the frequency. (B) Boxplot of the predicted cell type proportion. Boxes represent the $25^{\text {th }}$ and $75^{\text {th }}$ percentiles and internal line represents the median. The whiskers represent 1.5 multiplied by the inter-quartile range. Outliers are shown as individual points. 
Supplementary Figure 17. Cell type mediated eQTLs in cerebellum are mostly mediated by astrocytes and macrophages. The number of cell type interacting eQTLs for cerebellum deconvoluted cell types. We did not identify eQTLs that were shared between cell types. in this plot represents a comparison between bulk RNA-seq (y-axis) and single-nucleus RNA-seq (x-axis). Each dot represents one cis-eQTL, and the legend shows the Pearson correlation coefficient. Each column is a comparison between equivalent (and where not possible; similar) cell types in both datasets. Each row illustrates a different filtering on which eQTLs are shown and/or a different value on the y-axis. The x-axis always denotes the overall z-score of the eQTL effect in the single nucleus dataset of that respective column. (A) Meta-analysis eQTL z-score (y-axis) in Cortex-EUR bulk RNA-seq data, no filtering is applied. (B) Meta-analysis eQTL zscore (y-axis) in Cortex-EUR bulk data, eQTLs are filtered based on the Decon-QTL BenjaminiHochberg corrected p-value $<0.05$ in each respective column. (C) same as row B but now showing the log betas of the interaction model on the y-axis. (D) Meta-analysis eQTL z-score (yaxis) in bulk data for eQTLs that are significantly replicating in each respective dataset. Dots are colored if they are significantly cell type mediated (BH FDR <0.05) by the respective cell type in bulk data. (E) y-axis shows the log betas of the interaction model (y-axis) and filtering eQTLs on both significantly replicating in each respective dataset, as well as being significantly cell type

\section{Supplementary Figure 19. Bulk interacting eQTLs replicating in single-nucleus ROSMAP.} Replication of cell type interaction eQTLs for STMN4 (A), FAM221A (B), NKAIN1 (C) and SCL25A27 (D). First column: Boxplots of the eQTL effect in Cortex-EUR bulk RNA-seq. Second column: Cell type interacting eQTL effect in Cortex-EUR bulk RNA-seq. The X-axis shows the estimated cell type proportion, the $y$-axis shows the gene expression, each dot represents a sample, and the colors indicate the SNP genotype, with yellow being the minor allele. Values under the alleles are Spearman correlation coefficients. Third column: Forest plot of the spearman coefficient with effect direction relative to the minor allele when replicating the eQTL effect in ROSMAP single nucleus data $(n=38)$. Error bars indicate $95 \%$ confidence interval. Each row denotes a cell type specific dataset: excitatory neurons (EX), oligodendrocytes (OLI), inhibitory neurons (IN), astrocytes (AST), oligodendrocyte precursor cells (OPC), microglia (MIC), pericytes (PER) and endothelial cells (END). The bold cell type corresponds to the cell type that showed an interaction effect in bulk RNA-seq. Fourth column: Cell type interacting eQTL effect in ROSMAP single-nucleus RNA-seq $(n=38)$ of the bold highlighted cell

1542 Supplementary Figure 20. Mendelian Randomization summary. Each plot is for a different 1543 trait (Intelligence, Intracranial volume, Putamen volume, Years of schooling, Alzheimer's 1544 disease, Amyotrophic Lateral Sclerosis, Depression (broad), Frontotemporal Dementia, 
Parkinson's disease, Bipolar disorder, Generalized epilepsy, juvenile myoclonic epilepsy, multiple sclerosis and schizophrenia). For each SNP the effect allele (EA) is given, the eQTL beta of the EA on the given gene, the odds ratio (disease traits) or beta (quantitative traits) of the EA on the phenotype, and the Wald ratio p-value of the mendelian randomization analysis.

Supplementary Figure 21. Colocalization regional plots for five suggestive MR findings in made for five MR findings (CASS4 for Alzheimer's disease, TMEM170B for intelligence, GATAD2A for schizophrenia and years of schooling, and ZCWPW1 for years of schooling) in Cortex-EUR (top), eQTLGen (middle) and outcome GWAS (bottom) to show colocalization. These five findings all passed suggestive threshold $\left(\mathrm{p}<5 \times 10^{-5}\right)$ in Cortex-EUR, with eQTL effects replicated in eQTLGen $(\mathrm{p}<0.05)$, showed colocalization for both Cortex-EUR and eQTLGen but opposite directions of effect.

Supplementary Figure 22. Colocalization regional plots for two suggestive MR findings for multiple sclerosis that showed opposite directions of effect between Cortex-EUR and eQTLGen. Regional plots were made for two suggestive MR findings for MS (KMT5A, $R N F 19 B)$, both of which were suggestive signals in Cortex-EUR as well as eQTLGen ( $<<5 \times 10^{-}$ colocalization was only found in Cortex-EUR. WR comparison on the same gene but with the different SNP instruments selected by each study (matching on the top WR finding if gene instrumented with multiple SNPs in the study) and the bottom panel the WR comparison between MetaBrain instruments and eQTLGen matching on both the same gene and SNP instrument. Genes which showed opposite direction of WR effect between MetaBrain and eQTLGen are colored in red and the genes with the same direction in blue. GTEx for 28 multiple sclerosis genes for which there are no significant eQTLgen instruments in brain and blood. 
1582 predicted cell proportion for one sample. Numbers under the boxplots indicate the number of 1583 samples plotted. Values above the line are p-values from a t-test between groups.

Supplementary Figure 26. Forest plots for rs1990622 trans-eQTLs. Forest plots for each of the trans-eQTL genes associated with rs1990622. Each plot shows the trans-eQTL beta and 95\% confidence interval for each of the included datasets and the meta-analysis. Effect directions are relative to the A allele of rs1990622. Sizes of dots are relative to sample size of each dataset. Trans-eQTL effects are most pronounced in AMP-AD datasets.

Supplementary Figure 27. Summary of 7p21.3 locus trans-eQTLs. (A) Forest plots showing effect sizes for rs1990622 (yellow; beta and 95\% confidence interval) for cis-eQTL gene THSD7A, trans-eQTL gene CALB2, and association of rs1990622 with estimated neuron proportion. Right panel shows average estimated neuron proportions per dataset (blue violin plots). EQTL and neuron proportion associations are most pronounced in AMP-AD datasets, while average neuron proportions are comparable. (B) Trans-eQTL meta-analysis Z-scores for rs11974335, rs10950398 and rs1990622 (x-axis), and the correlation of those trans-eQTL genes with predicted neuron proportion (y-axis) are highly correlated. (C) Comparison of trans-eQTL Z-scores between Alzheimer's disease patients (x-axis) and neurotypical controls (y-axis) shows

Supplementary Figure 28. Replication of cortex trans-eQTLs in single-nucleus data. Each figure in this plot represents a comparison between bulk RNA-seq (y-axis) and single-nucleus RNA-seq (x-axis). Each dot represents one trans-eQTL, and the legend shows the Pearson correlation coefficient. Each column is a comparison between equivalent (and where not possible; similar) cell types in both datasets. Each row illustrates a different filtering on which eQTLs are shown and/or a different value on the y-axis. The X-axis always denotes the overall zscore of the eQTL effect in the single nucleus dataset of that respective column. (A) Metaanalysis eQTL z-score (y-axis) in Cortex-EUR bulk RNA-seq data, no filtering is applied. (B) Meta-analysis eQTL z-score (y-axis) in Cortex-EUR bulk data, eQTLs are filtered based on the Decon-QTL Benjamini-Hochberg corrected p-value $<0.05$ in each respective column. (C) same as row $\mathbf{B}$ but now showing the log betas of the interaction model on the y-axis. (D) Meta-analysis eQTL z-score (y-axis) in bulk data for eQTLs that are significantly replicating in each respective dataset. Dots are colored if they are significantly cell type mediated (BH FDR $<0.05$ ) by the respective cell type in bulk data. (E) y-axis shows the log betas of the interaction model (y-axis) and filtering eQTLs on both significantly replicating in each respective dataset, as well as being 
each gene derived from a leave-one-out procedure. One of the parameters to build the network is the number of eigenvectors to use after PCA over the gene correlation matrix. Here we show for the 6 annotation categories (KEGG, REACTOME, GO Biological Process, GO Molecular Function, GO Cellular Component, and HPO) the AUC mean (dot) and standard deviation (lines) at different eigenvector cut-offs. The red dot and line indicate the eigenvector cut-off that was used for that annotation category.

Supplementary Figure 30. Heatmaps of the Pearson correlation of the AUC values between different eigenvector cut-offs. Correlation was calculated between the different eigenvector cutoffs for the 6 annotation categories.

Supplementary Figure 31. (A) UMAP representation of heterogeneous gene network. Immune and blood cell types show increased gene expression levels for genes prioritized using Downstreamer for multiple sclerosis, while decreased expression is observed in brain related tissues. (B) Within MetaBrain, those same genes show lower expression in cortex, but higher expression in spinal cord and cerebellum.

Supplementary Figure 32. Spearman correlation heatmap of predicted cell fractions versus principal components calculated using all MetaBrain samples. A heatmap showing the first fifty principal components as the columns and the five cell types for which we predicted proportions as rows. Each cell is colored based on the spearman correlation coefficients. Blue denotes a negative correlation, red a positive correlation and white denotes no correlation. reduction plot of 39 snRNA-seq samples from ROSMAP. Each dot represents a single cell $(n=70,634)$. The dots are colored by their corresponding cell type: excitatory neurons $(\mathrm{EX})$, oligodendrocytes (OLI), inhibitory neurons (IN), astrocytes (AST), oligodendrocyte precursor cells (OPC), microglia (MIC), pericytes (PER) and endothelial cells (END). reduction plot of 39 snRNA-seq samples from ROSMAP. Each dot represents a single cell $(n=70,634)$. The dots are colored by their corresponding cell type subcluster: excitatory neurons (EX), oligodendrocytes (OLI), inhibitory neurons (IN), astrocytes (AST), oligodendrocyte 


\section{Table descriptions}

1658 Table 1. Prioritized genes from the Mendelian Randomization analysis on MetaBrain

1659 eQTLs versus brain related outcomes. Harmonized eQTL and GWAS SNP effects and single

1660 SNP Wald Ratio estimates are reported in the table for all genes with Wald Ratio effects at

$1661 \mathrm{P}<1.865 \times 10^{-7}$. Columns are genomic position, rsid and alleles for SNP instrument (EA: Effect

1662 allele. NONEA: non-effect allele. proxy SNP: rsid of proxy SNP replacement used for outcome

1663 if instrument not present in GWAS), the SNP effects (beta, SE, p) for the MetaBrain eQTLs

1664 followed by the SNP effects for the brain related outcomes and then the Wald Ratio effects. 


\section{Supplementary Table descriptions}

\section{Supplementary table 1. Number of samples and individuals.} Sheet Genotype QC: The number of genotype individuals and samples pre-QC (column C-H) and post-QC (column I-N) for the different RNA-seq (column A) and genotype (column B) datasets. Columns are: PreQC: Number of initial genotype samples processed for QC. PostQC: Number of genotype samples left after QC filtering. RNA-seq dataset: Name of the complete dataset. Genotype dataset: Name of the genotype dataset. Some datasets have multiple genotype platforms, or multiple smaller datasets that are part of the larger RNA-seq dataset. Individuals: The number of individuals per dataset. EUR: Number of genotype samples per dataset of individuals of European population. AFR: Number of genotype samples per dataset of individuals of African population. EAS: Number of genotype samples per dataset of individuals of East-Asian population. SAS: Number of genotype samples per dataset of individuals of SouthAsian population. AMR: Number of genotype samples per dataset of individuals of Ad Mixed American population. Sheet RNA-QC: The number of RNA-seq samples at different steps of QC and for different brain regions. Cells A2-F18 have the number of samples at different QC steps. Columns are: Dataset: dataset name. Number of RNA-seq samples: Number of RNA-seq samples processed to go through QC. Alignment QC: Number of RNA-seq samples left after filtering on alignment QC (e.g. percent reads aligned). RNA-seq PCA outliers - step 1: Number of RNA-seq samples left after filtering samples >4SD from mean of PC1. RNA-seq PCA outliers - step 2: Number of samples left after recalculating PCA and again removing samples >4SD fom mean of PC1. Covariate removal: Number of samples left after covariate removal. RNA Tissue grouping: the meta-data across different datasets uses different granularity of tissue annotation. Tissues were grouped accordingly.

Sheet Sample Links: RNA-seq samples linked to genotype samples. Left top: numbers of RNAseq sample linked to a genotype sample per dataset, per population. Top right: number of unique individuals per dataset per population. Middle: number of uniquely linked individuals per dataset, per population and per tissue group. Bottom: numbers of individuals used from each

\section{Supplementary table 2. Cis-eQTL summary statistics.}

Cis-eQTL summary statistics listing index variant per gene (FDR $<0.05)$. One sheet per eQTL discovery dataset. Genomic positions are GRCh38. eQTL Rank: whether the eQTL is a primary, secondary, tertiary, quaternary, or higher eQTL.

Supplementary table 3. Number of cis- and trans-eQTLs. For each dataset the number of cisand trans-eQTL SNPs, genes, and SNP-gene combinations found at FDR $<0.05$. Columns are:

1703 Basalganglia, Cerebellum, Cortex, Hippocampus, Spinalcord: the five different brain regions for which eQTL calling was done. EUR: Number of eQTLs with samples from European

1705 population. AFR: Number of eQTLs with samples from African population. EAS: Number of 
eQTLs with samples from East-Asian population. EUR+AFR, wo ENA, no PCA: Number of eQTLs with samples from EUR and AFR populations, excluding samples from the ENA cohorts, and using gene expression levels that were not corrected for principal components. rank eQTLs. Gene set enrichment summary statistics generated using g:Profiler for genes having a primary eQTL effect (sheet Primary eQTL), and those also having a secondary eQTL

1713 (sheet Non-primary eQTL).

Supplementary table 5. Gene set enrichment summary statistics generated using g:Profiler for genes having an eQTL effect in cerebellum.

Supplementary table 6. GTEx cis-eQTL replication. Replication between cis-eQTLs of different MetaBrain regions and all GTEx tissues. Discovery was performed in each MetaBrain dataset while excluding GTEx, and then replicated in each GTEx tissue. Tested eQTLs: those eQTLs that were also present in the GTEx dataset. Proportion shared and FDR<0.05: proportion of tested eQTLs that was also significant in GTEx. Concordant and FDR<0.05: number of tested eQTLs that was also significant and for which the allelic direction was concordant. Concordance: proportion of concordant tested and significant eQTLs. discovery cohort and eQTLgen eQTLs as replication cohort. Top table: FDR $<0.05$ in MetaBrain discovery only (FDR $<1$ in eQTLGen). Bottom table: FDR $<0.05$ in both MetaBrain and eQTLgen datasets. Shared: number of shared eQTLs. Concordant: number of shared eQTLs that has the same allelic direction of effect. Concordant over total: proportion of concordant eQTLs over the total number of eQTLs discovered. Concordant over shared: proportion of concordant eQTLs over number of shared eQTLs. Decon-eQTL results for cortex. Sheet cerebellum: All Decon-eQTL results for cerebellum. Columns for both sheets are: Gene: deconvoluted eQTL gene ensebl ID. Gene symbol: deconvolution eQTL gene symbol. SNP: deconvoluted eQTL SNP. Alleles: SNP alleles. Effect Allele: the allele to which the betas are directed. Columns ending with p-value: $p$-value for the cell-type interaction. Columns ending with beta: beta for the cell-type proportion term. 
1742 Supplementary table 9 Replication of the MetaBrain cortex primary cis-ieQTLs in

1743 ROSMAP single-nucleus data. For each of the deconvoluted cell-types, the FDR and betas are

1744 listed. For each of the cell types in the single nucleus data, the FDR and eQTL Z-scores are

1745 listed. All betas andZ-scores are relative to the Effect Allele.

1747 Supplementary table 10. eQTL SNPs in linkage disequilibrium with GWAS SNPs. The

1748 GWAS SNPs that are in high linkage disequilibrium (LD) with the cis-eQTL SNPs. Each sheet is

1749 a different metabrain eQTL datasets from EUR populations. The sheet Included Traits lists

1750 GWAS traits that were tested. Columns are: eQTL rank: the rank of conditional eQTLs

1751 (1=primary, 2=secondary, etc). GWASID: GWAS ID of the GWAS SNP. Trait: Name of the

1752 GWAS trait. Index variant: the GWAS variant. Index Variant P: GWAS p-value. Index

1753 Variant Alleles: Alleles of the GWAS variant. Index Variant Effect: GWAS effect. Linked

1754 EQTL SNP: the eQTL SNP. LD(rsq): the LD $\mathrm{r}^{2}$. LinkedEQTLGenes: the eQTL genes that the

1755 linked SNP affects. Linked EQTL Gene Symbols: HGNC name of the linked genes. Linked

1756 EQTL Alleles: Alleles of the eQTL SNP. Linked EQTL Effect Allele: The allele that is related

1757 to the effect direction. Linked EQTL Zscores: Z-scores of the eQTL effect. Linked EQTL P:

1758 p-value of the eQTL effect. GWAS Cluster Size: Number of GWAS SNPs in LD with Index

1759 Variant. SNPs In GWAS Cluster: SNPs that are in LD with the Index Variant.

Supplementary table 11. List of traits used in Mendelian randomization and colocalization

1764 Supplementary table 12 eQTL SNPs which showed evidence of genetic colocalization with tested brain-related traits. ID, Chromosome, Position, SNP, Effect Allele, Non Effect Allele: Position of instrumenting SNP with effect allele used during the harmonization procedure. Proxy used, Proxy SNP: whether proxy lookup had to be performed to find SNP in outcome GWAS and the rsid of the proxy used. MetaBrain SNP effects: gene name and summary statistics for the instrument-exposure SNP association (MetaBrain eQTL). Outcome SNP effects: outcome name (neurological trait) and summary statistics for the harmonized instrument-outcome SNP association. MR effects: single SNP Wald ratio effect between the instrumented eQTL and neurological outcome. Coloc results: colocalization probability of both traits sharing the same causal variant in the region. Decon-QTL results: eQTL SNP: the SNP that was tested for cell type mediated effects. In some cases a SNP which is in high LD with the instrument SNP is used for Decon-QTL. LD R-squared: the LD between SNP and eQTL SNP. Columns listing DeconQTL results: beta: the beta of the interaction term in the Decon-QTL model with respect to the Effect Allele column. FDR: the Benjamini-Hochberg corrected interaction p-value. Mendelian 

Cortex-EUR primary eQTLs (columns A to $\mathbf{P}$ were adapted from Schwartzentruber et al. for comparisons and columns $\mathbf{Q}$ to $\mathbf{Y}$ are MetaBrain findings. Category - 1: previously identified and replicated in MetaBrain Cortex-EUR, 2: novel results found by MetaBrain Cortex-EUR, 3: previously identified but not replicated in MetaBrain Cortex-EUR. different SNP instruments. For this analysis, the Wald Ratio effects for the top hit eQTL for each gene within each study were compared. (b) Wald Ratio comparison on the same gene fixing on the same eQTL instrument between studies. For this analysis, the eQTLGen Wald Ratios were re-derived using the second Taylor expansion error term on the same SNP instruments as MetaBrain. discordance. This table displays the colocalization results for 31 suggestive MR findings from Cortex-EUR with eQTL instruments replicated in eQTLGen $(\mathrm{p}<0.05)$ but allelic discordance (opposite directionalities of alleles). Highlighted rows are findings with colocalization in both Cortex-EUR and eQTLGen.

Supplementary table 16. Comparison of MR suggestive hits for MS between metaBrain and eQTLGen. This table displays 157 suggestive MR signals for multiple sclerosis in CortexEUR and the replication MR and colocalization results of corresponding genes in eQTLGen. eQTLs detected in this study (FDR<0.05). Percentage cross-mapping: percentage of the gene that can be mapped within $5 \mathrm{Mb}$ of the trans-eQTL SNP. Sheet Trans-eQTLs no crossmap: transeQTLs that remain significant after cross-mapping eQTLs have been removed. Sheet TranseQTLs with cis per trait: in this sheet, trans-eQTLs are annotated with cis-eQTLs for the same SNP, and subsequently split per trait annotation for the SNP. Consequently, a single trans-eQTL may be represented by multiple rows. Sheet Convergent trans-eQTLs: genes on which multiple independent loci have a trans-eQTL, split per annotated trait. Sheet TraitsAndNrOfSNPs: list of cell-type proportions. Sheet Cortex-EUR: associations (FDR $<0.05)$ while limiting to Cortex- 

datasets. T-test $\mathrm{p}$-values comparing neuron proportions for pairwise comparisons between the datasets included in the trans-eQTL analysis. Trans-eQTL Z-scores for three SNPs (rs11974335, rs10950398, and rs1990622), and correlations of the trans-eQTL genes with predicted neuron proportions. genes that show downregulation due to the 7p21.3 trans-eQTL effect alleles. Sheet upregulated genes: gene set enrichments for genes that show upregulation due to the $7 \mathrm{p} 21.3$ trans-eQTL effect alleles. ROSMAP single-nucleus data. For each of the deconvoluted cell-types, the FDR and betas are listed. For each of the cell types in the single nucleus data, the FDR and eQTL Z-scores are listed. All betas and Z-scores are relative to the Effect Allele.

Supplementary table 23. Downstreamer results for amyotrophic lateral sclerosis in EUR samples. GenePrioritization_MetaBrainCerebellumOnly: gene prioritization performed in MetaBrain cerebellum samples. Sheets Reactome_MetaBrain, GO_BP_MetaBrain, GO_CC_MetaBrain, GO_MF_MetaBrain, KEGG_MetaBrain, and HPO_MetaBrain: gene set enrichments for coregulated genes identified using Downstreamer. Sheets Expression_MetaBrain, Expression_HCA, and GtexV8_relative: expression enrichment using all lists set of ontologies tested for this phenotype. Sheet GenePrioritization_MetaBrain: gene prioritization performed in all MetaBrain samples. Sheet

1853 GenePrioritization_MetaBrainCortexOnly: gene prioritization performed in MetaBrain cortex 1854 samples. GenePrioritization_MetaBrainCerebellumOnly: gene prioritization performed in MetaBrain cerebellum samples. Sheets Reactome_MetaBrain, GO_BP_MetaBrain, 
GO_CC_MetaBrain, GO_MF_MetaBrain, KEGG_MetaBrain, and HPO_MetaBrain: gene set enrichments for coregulated genes identified using Downstreamer. Sheets Expression_MetaBrain, Expression_HCA, and GtexV8_relative: expression enrichment using all MetaBrain samples, Human Cell Atlas, and GTEx v8.

Supplementary table 25. Downstreamer results for schizophrenia. Sheet overview: lists set of ontologies tested for this phenotype. Sheet GenePrioritization_MetaBrain: gene prioritization performed in all MetaBrain samples. Sheet GenePrioritization_MetaBrainCortexOnly: gene prioritization performed in MetaBrain cortex samples. GenePrioritization_MetaBrainCerebellumOnly: gene prioritization performed in MetaBrain cerebellum samples. Sheets Reactome_MetaBrain, GO_BP_MetaBrain, GO_CC_MetaBrain, GO_MF_MetaBrain, KEGG_MetaBrain, and HPO_MetaBrain: gene set enrichments for coregulated genes identified using Downstreamer. Sheets Expression_MetaBrain, Expression_HCA, and GtexV8_relative: expression enrichment using all MetaBrain samples, Human Cell Atlas, and GTEx v8.

Supplementary table 26. Downstreamer results for Alzheimer's disease. Sheet overview: lists set of ontologies tested for this phenotype. Sheet GenePrioritization_MetaBrain: gene prioritization performed in all MetaBrain samples. Sheet GenePrioritization_MetaBrainCortexOnly: gene prioritization performed in MetaBrain cortex samples. GenePrioritization_MetaBrainCerebellumOnly: gene prioritization performed in MetaBrain cerebellum samples. Sheets Reactome_MetaBrain, GO_BP_MetaBrain, GO_CC_MetaBrain, GO_MF_MetaBrain, KEGG_MetaBrain, and HPO_MetaBrain: gene set enrichments for coregulated genes identified using Downstreamer. Sheets Expression_MetaBrain, Expression_HCA, and GtexV8_relative: expression enrichment using all

Supplementary table 27. Downstreamer results for multiple sclerosis. Sheet overview: lists set of ontologies tested for this phenotype. Sheet GenePrioritization_MetaBrain: gene prioritization performed in all MetaBrain samples. Sheet GenePrioritization_MetaBrainCortexOnly: gene prioritization performed in MetaBrain cortex samples. GenePrioritization_MetaBrainCerebellumOnly: gene prioritization performed in MetaBrain cerebellum samples. Sheets Reactome_MetaBrain, GO_BP_MetaBrain, GO_CC_MetaBrain, GO_MF_MetaBrain, KEGG_MetaBrain, and HPO_MetaBrain: gene set enrichments for coregulated genes identified using Downstreamer. Sheets Expression_MetaBrain, Expression_HCA, and GtexV8_relative: expression enrichment using all 
1894 Supplementary table 28. Downstreamer results for amyotrophic lateral sclerosis in EUR

1895 population. Sheet overview: lists set of ontologies tested for this phenotype. Sheet

1896 GenePrioritization_MetaBrain: gene prioritization performed in all MetaBrain samples. Sheet

1897 GenePrioritization_MetaBrainCortexOnly: gene prioritization performed in MetaBrain cortex

1898 samples. GenePrioritization_MetaBrainCerebellumOnly: gene prioritization performed in

1899 MetaBrain cerebellum samples. Sheets Reactome_MetaBrain, GO_BP_MetaBrain,

1900 GO_CC_MetaBrain, GO_MF_MetaBrain, KEGG_MetaBrain, and HPO_MetaBrain: gene set

1901 enrichments for coregulated genes identified using Downstreamer. Sheets

1902 Expression_MetaBrain, Expression_HCA, and GtexV8_relative: expression enrichment using all

1903 MetaBrain samples, Human Cell Atlas, and GTEx v8.

1904

1905 Supplementary table 29. ENA accession IDs. List of study accession IDs collected from

1906 European Nucleotide Archive. Columns are: study_accession: ID of the study in ENA.

1907 run_accession: ID of all the ENA runs included in this study (before quality control) 


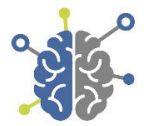

15 datasets

6,518 individuals

8,727 RNA-seq samples

MetaBrain

\section{7 brain regions}

Hypothalamus

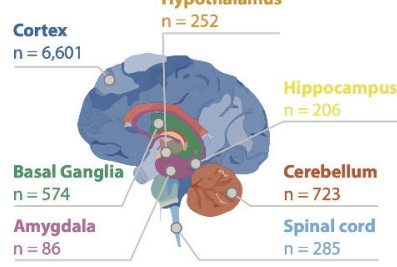

\section{Cis-eQTLs}

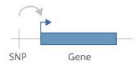

27,226 primary cis-eQTLs

\section{Secondary eQTLs}

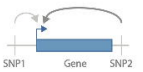

16,192 non-primary cis-eQTLs

Trans-eQTLs

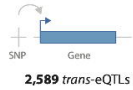

Interaction eQTLs

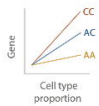

1,515 interaction eQTLs
Mendelian randomization and colocalization
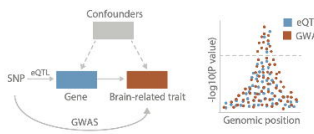

$\mathbf{4 3 9}$ prioritized genes for $\mathbf{2 7}$ brain related traits

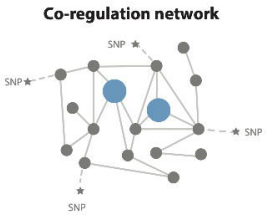

208 genes prioritized for $\mathbf{5}$ traits 
A

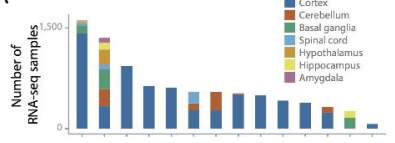

B

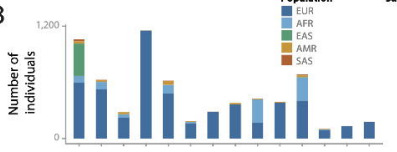

C

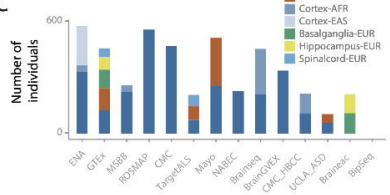

D

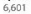

6,601
723

574

285
252

206

sample size

5,138

805

351
170

52

ஸ̃

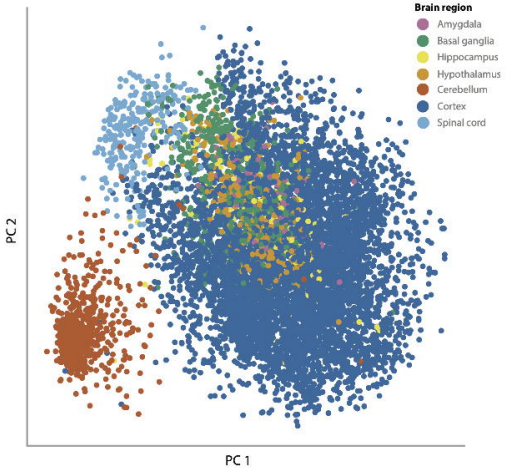


$A$

\section{A Cerebellum}

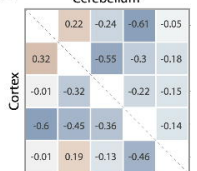

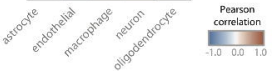

D

STMN4

Cortex RNA-seq

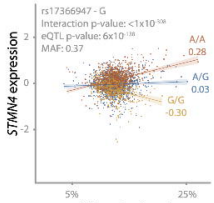

Oligodendrocyte proportion

astrocyte

endothelial

macrophage

neuron

oligodendrocyte

Pearson

(a)

(1)

B

E

SnRNA-seq

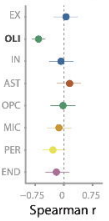

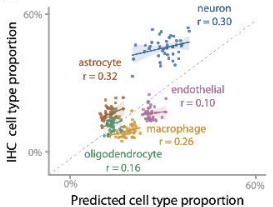

Predicted cell type proportion
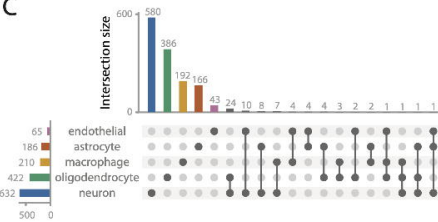

F

snRNA-seq

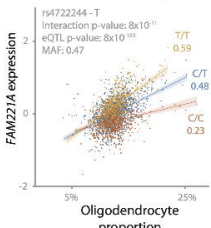

proportion

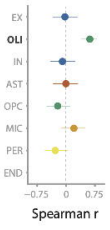

SnRNA-seq

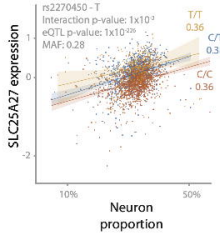

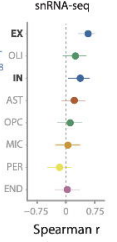




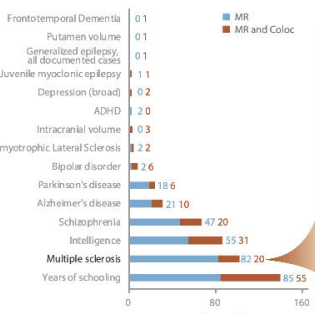

Number of loci

\section{SNP - EA}

1562120372-A

r52288481-A

rs9865116-G

rs9970196-T

Ts344811 144 - T

rs180534-C

rs6980563-C

157306304-A

ris10877011--

T52259735-C

rs11177335-T

153309527-A

r54796224-G

ז59783655 - T

5131813 - A

rs6421983-T

r54794000 - G

rs1 19877023 - A

154578918- $T$

rs28995017-A

\section{Gene}

MPYi7.2

coders5

MYNN

TTC3.

RNAT

CIEQ?

EEFIAKMTS

CYP24A?

TSPX
TMOIO
TRAF3

MTO

SCO2
IFTMM!
HOSEP?

MPEPPS

'SSPAN3!

SLC12AS

HLADDR,

FLADRA

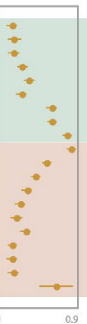

eQTL beta

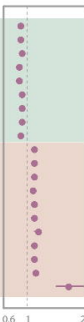

Multiple sclerosis OR
$7.3 \times 10^{7}$

$9.3 \times 10 \times 10$

$7.2010^{*}$

$20 \times 10^{15}$

$2.3 \times 10^{\circ}$

$2.1 \times 10^{\circ}=$

$17 \times 10^{2}$

$7.6 \times 10^{\circ}$

$5.1 \times 10^{-11}$

$7.7 \times 10$ :

7..5x19

$3,2 \times 10^{8}$

$1.6 \times 10^{7}$

$1.0 \times 10^{8}$

$6.2 \times 10^{-0}$

$7.8 \times 10^{-5}$

$3.0 \times 10^{\circ}$

$17 \times 190^{\circ}$

$7,9 \times 10^{\circ}$

$1.4 \times 10^{13}$

Wald ratio P-value

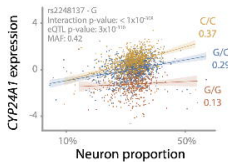

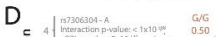

듬

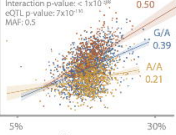

Macrophage proportion 
A

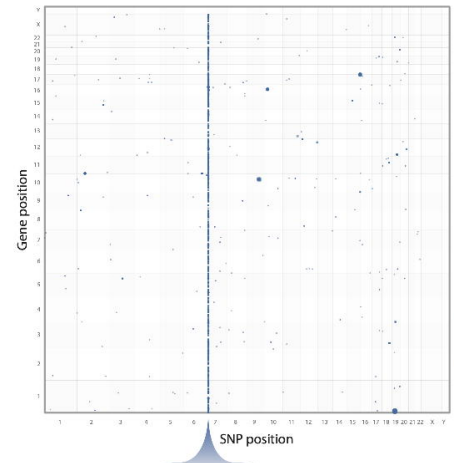

B

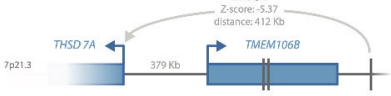

SNP:

Number of datosets:

Upregulated trans-eQTL genes:

rs1 1974335-G rs10950398-G rs1990622-A

$\begin{array}{cc}4 & 6 \\ 275 & 200 \\ 750 & 590\end{array}$

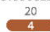

Downregulated trans-eQTL genes:

750

200

590

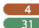

31

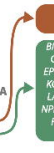

C

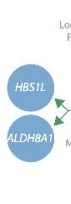

\section{$2 p 16.1$}

xated $h$ intron of $B C 11 \mathrm{~A}$ Fetal hemoglchin levels:

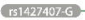

.54895447:G

$$
6923.3
$$

Mean corpuscular volume

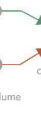

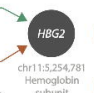

subunit

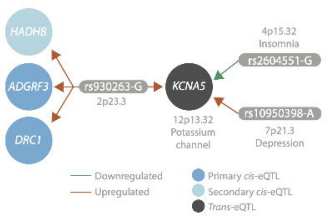

2,252 trans-eQTLs

in 7 p21.3 locus 
A Genes, co-regulated with

genes inside ALS loci:

MetaBrain

cerebellum

All MetaBrain samples samples

MAPKSIPZ

DNAUCS

SICAA3

MGAT3

UBRa

SMG6 Clorf69

SMGS HIFTAN VEATS

PRRCZB GRAMDIB USP2O

ARP12 G6PD MED22

TSCI FLYWCHI FAMI3AC

SEQATC HIT ATPGVOA
ZEAT RPTOR VACTA

MetaBrain

cortex samples
B

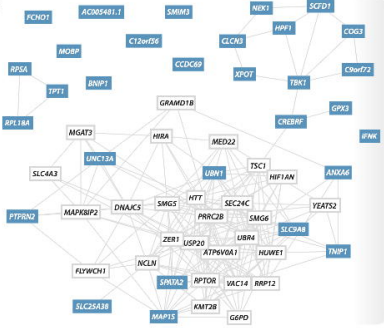

C

\section{Associated phenotypes:}

p-value HPOenrichments

$6.69 \times 10^{-15} \quad$ Cerebral visual impairment

$1.24 \times 10^{-7}$ Galt disturbance

$1.33 \times 10^{-7}$ Cerebral cortical atrophy

$2.17 \times 10^{-17}$ Cerebellar atrophy

$2.94 \times 10$ : 7 A.bnormality of the cerebral white matter

Gene inside ALS locus $\square$ Gene, co-regulated with genes inside ALS loci

\section{Genes, co-regulated with genes inside MS loci:}

\section{Heterogeneous \\ co-regulation \\ network \\ All MetaBrain \\ samples}

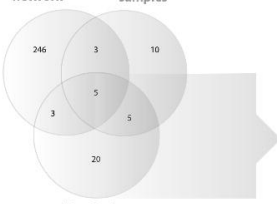

MetaBrain

cortex samples
E

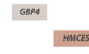

$$
\text { DKLA }
$$$$
\text { HMCES }
$$

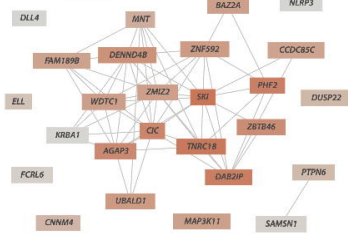

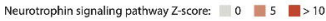

$\mathbf{F}$

\section{Associated pathways:}

p-value KEGGenrichments

$4.8 \times 10^{-15}$ Aeute myaloid leukomia

$8.3 \times 10^{-17}$ T cell receptor signaling pathway

$1.6 \times 10^{-15} \quad$ Chemokine signaling pathway

$1.4 \times 10^{-2}$ Neurotrophin signaling pethway

$5.7 \times 10^{14}$ Endocytosis 